\title{
Neural responses to emotional expression information in high- and low-spatial frequency in autism: evidence for a cortical dysfunction
}

\author{
Corrado Corradi-Dell'Acqua ${ }^{1,2 *}$, Sophie Schwartz ${ }^{2}$, Emilie Meaux ${ }^{2}$, Bénedicte Hubert ${ }^{3,4}$, \\ Patrik Vuilleumier $^{1,2}$ and Christine Deruelle ${ }^{4}$ \\ 1 Swiss Center for Affective Sciences, University of Geneva, Geneva, Switzerland \\ ${ }^{2}$ Laboratory for Neurology and Imaging of Cognition, Department of Neuroscience and Clinic of Neurology, University Medical Center, Geneva, Switzerland \\ ${ }^{3}$ Hôpital Rivière-de-Praires, University of Montréal, Montréal, OC, Canada \\ ${ }^{4}$ CNRS, Institut de Neurosciences de la Timone, Aix-Marseille Université, Marseille, France
}

Edited by:

Leonie Koban, University of

Colorado Boulder, USA

\section{Reviewed by:}

Nathalie George, Centre National de la Recherche Scientifique, France Martin Schulte-Rüther, Universityl Hospital RWTH Aachen, Germany

\section{${ }^{*}$ Correspondence:}

Corrado Corradi-Dell'Acqua, Swiss Center for Affective Sciences, NCCR Affective Sciences, University of Geneva - CISA, Campus Biotech, Uni Durfour, 24 rue Géneral Dufour, CH-1211 Geneva, Switzerland e-mail: corrado.corradi@unige.ch
Despite an overall consensus that Autism Spectrum Disorder (ASD) entails atypical processing of human faces and emotional expressions, the role of neural structures involved in early facial processing remains unresolved. An influential model for the neurotypical brain suggests that face processing in the fusiform gyrus and the amygdala is based on both high-spatial frequency (HSF) information carried by a parvocellular pathway, and low-spatial frequency (LSF) information separately conveyed by a magnocellular pathway. Here, we tested the fusiform gyrus and amygdala sensitivity to emotional face information conveyed by these distinct pathways in ASD individuals (and matched Controls). During functional Magnetical Resonance Imaging (fMRI), participants reported the apparent gender of hybrid face stimuli, made by merging two different faces (one in LSF and the other in HSF), out of which one displayed an emotional expression (fearful or happy) and the other was neutral. Controls exhibited increased fusiform activity to hybrid faces with an emotional expression (relative to hybrids composed only with neutral faces), regardless of whether this was conveyed by LSFs or HSFs in hybrid stimuli. ASD individuals showed intact fusiform response to LSF, but not HSF, expressions. Furthermore, the amygdala (and the ventral occipital cortex) was more sensitive to HSF than LSF expressions in Controls, but exhibited an opposite preference in ASD. Our data suggest spared LSF face processing in ASD, while cortical analysis of HSF expression cues appears affected. These findings converge with recent accounts suggesting that ASD might be characterized by a difficulty in integrating multiple local information and cause global processing troubles unexplained by losses in low spatial frequency inputs.

Keywords: autism, facial expression, emotion expression, spatial frequency, fMRI

\section{INTRODUCTION}

Autism Spectrum Disorder (ASD) is a pervasive neurodevelopmental disorder characterized by dysfunctional socialization and communication, with the emergence of stereotyped and repeated behavior. Although this disorder is mostly known for its social symptoms, a wealth of studies converge in reporting atypicalities in elementary aspects of perception, as in the case of visual processing of (emotional and neutral) facial expressions (see Harms et al., 2010; Gaigg, 2012; Weigelt et al., 2012, for meta-analyses and reviews).

A wealth of studies based on abstract and geometrical stimuli, suggest that in ASD individuals have difficulties in processing visual stimuli in a global fashion, focusing instead on details and local information (e.g., Dakin and Frith, 2005; Happé and Frith, 2006; Mottron et al., 2006). These accounts can potentially explain also ASD atypical processing of faces, especially considering that facial properties (identity, gender, emotional expressions, etc.) are not usually processed by the analysis of isolated local features, but of how all different features relate one another at the global level (configural processing). In this perspective, tasks asking neurotypical individuals to assess the sameness of two faces usually report poorer performance when the standard spatial relation between the parts is distorted, as for upside-down faces (Valentine, 1988), composites made of two aligned half-faces from different people (Young et al., 1987), or faces with scrambled parts (Tanaka and Farah, 1993). However, studies implementing the same tasks in individuals with ASD have reported mixed findings with some describing them as not influenced (Van Der Geest et al., 2002; Joseph and Tanaka, 2003; Teunisse and de Gelder, 2003; Rondan and Deruelle, 2004; Riby et al., 2009) or less influenced than Controls (Hobson et al., 1988; López et al., 2004; Barton et al., 2007; Pellicano et al., 2007, see also Weigelt et al., 2012), but others describing equal effects as in neurotypical individuals (Teunisse and de Gelder, 2003; Rouse et al., 2004; Lahaie et al., 2006; Gross, 2008). Such variability could reflect the important heterogeneity of the ASD population, in which diagnostic symptoms are expressed differently across individuals, maybe confounded by age or attentional factors (Rondan 
and Deruelle, 2007), and/or possibly stem from the development of compensatory neuronal mechanisms (Gaigg, 2012; Dickstein et al., 2013).

To better characterize the face processing atypicalites observed in ASD, several studies have focused on the spatial frequency at which specific information is conveyed, suggesting that distinct frequencies might play different roles in face processing (Deruelle et al., 2004, 2008; Rondan and Deruelle, 2004; Boeschoten et al., 2007a; Vlamings et al., 2010). Indeed, local information can be processed only through high-spatial frequencies (HSF), whereas global configurations can be retained also from low spatial frequencies (LSF). It is well known that HSF visual information is carried by parvocellular pathways (see Figure 1, orange arrow) which reach the striate cortex and project almost exclusively to ventral occipito-temporal structures, including that part of the fusiform cortex which processes face stimuli (Fusiform Face Area [FFA], Kanwisher et al., 1997). LSF information instead is conveyed by magnocellular pathways (Figure 1, blue arrow) which project mostly to dorsal to parietal regions and, in less extent, to ventral cortical visual areas (Livingstone and Hubel, 1987, 1988). In addition, however, it has been proposed that the amygdala, a medial temporal structure critically involved in processing emotional expression in faces (Vuilleumier and Pourtois, 2007; Pessoa and Adolphs, 2010), may receive direct subcortical inputs from an additional collicular-pulvinar projection of magnocellular pathways (De Gelder et al., 1999; Morris et al., 1999), allowing the amygdala and ventral visual stream to receive coarse (LSF), but fast, information about facial emotional expressions (Vuilleumier et al., 2003, 2004; Winston et al., 2003b; Carretié et al., 2007; Vuilleumier and Pourtois, 2007). In this perspective, the frequent reports of atypical fusiform and/or amygdala responses to face stimuli in ASD (Baron-Cohen et al., 2000; Critchley et al., 2000; Schultz et al., 2000; Pierce et al., 2001; Hall et al., 2003; Hubl et al., 2003; Wang et al., 2004; Grelotti et al., 2005; Ashwin et al., 2007; Kleinhans et al., 2008; Scherf et al., 2010) raise the question of whether these effects might depend on differential visual frequency information conveyed by parvocellular (cortical) or magnocellular (also subcortical) pathways.

A number of studies have employed electrophysiological recording or behavioral techniques in children with ASD using high or low spatial filtered stimuli. While some results suggested that ASD affects preferentially the visual pathway conveying LSF (Deruelle et al., 2004, 2008; Boeschoten et al., 2007a; Vlamings et al., 2010), similar approaches in adults reported an ability to process LSF expressions comparable to that of neurotypical individuals (Rondan and Deruelle, 2004). To the best of our knowledge, no study has ever tested directly how, in ASD, the fusiform gyrus and the amygdala respond to HSF and LSF information in human faces.

In the present study, we showed to adults with ASD and matched Controls hybrid facial stimuli, which were generated according to a methodology used in previous studies (Schyns and Oliva, 1999; Winston et al., 2003b), by merging a HSF face with a LSF face of opposite gender (see Figure 2). These stimuli are particularly suited for our purpose as they offer to the observer both high- and low- extremes of the frequency spectrum of faces in the same stimulus, allowing us to determine

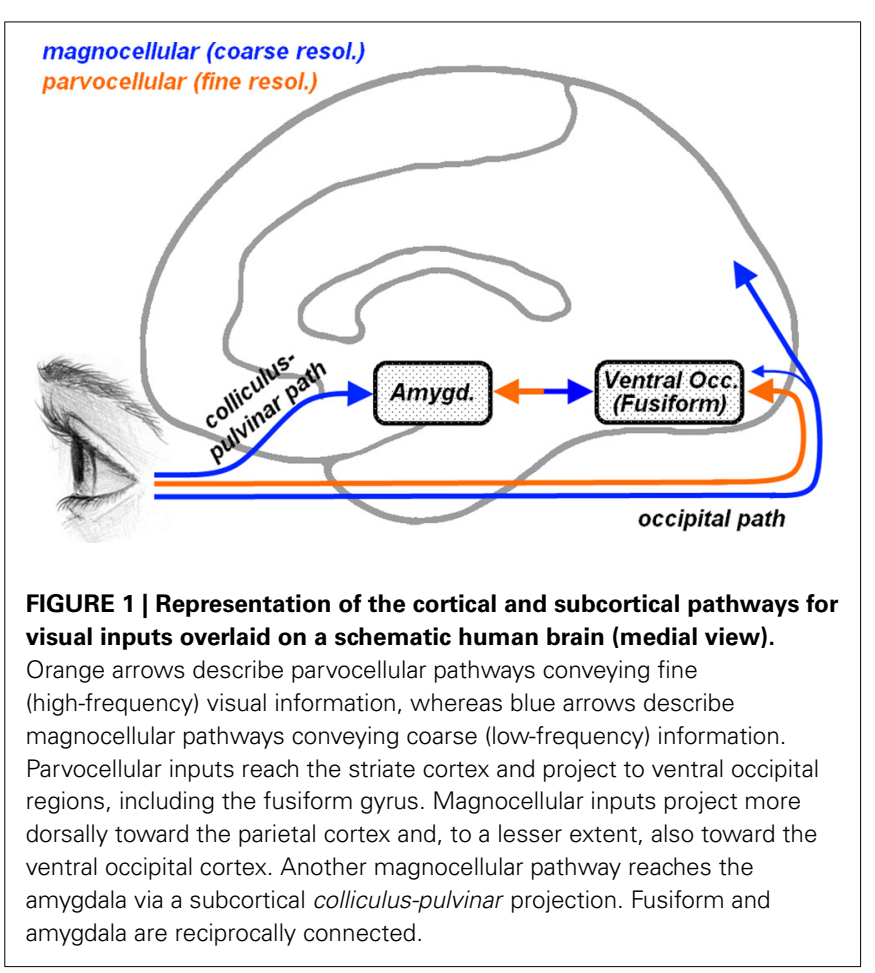

the band in which specific information is preferentially selected for face processing and responded to in different brain areas. Critically, in addition to mixing opposite genders in each spatial frequency band, our hybrid stimuli were made of the combination of a neutral expression and an emotional expression, with the latter being either fearful or happy and contained either in the HSFs or LSFs (counterbalanced across the gender dimension). In order to probe for differential responses to emotion expressions triggered by one or the other frequency bands, we engaged our participants in a gender discrimination task in which they had to report the apparent gender of each face. In a separate condition, our participants instead had to watch passively each stimulus, allowing us to determine any influence of different task demands. This led to a factorial design with group (ASD participants, Controls), frequency (emotional expressions conveyed by HSF, LSF), valence (fearful, happy expressions), and task (gender discrimination, passive viewing). For high-level baseline, we used (in each task) hybrid stimuli with no emotional expression (i.e., mix of neutral female and neutral male). Following previous studies on neurotypical individuals, we expected increased activity in ventral visual cortex (including the fusiform gyrus) when Controls discriminated faces in which emotions were conveyed by either HSFs or LSFs (as opposed to neutral expressions), as evidence of parvocellular cortical and magnocellular visual inputs respectively (Vuilleumier et al., 2001, 2003; Winston et al., 2003b; Rotshtein et al., 2007). The critical question, however, was whether these parvo- and magnocellular neural signatures were observable also in individuals with ASD. We reasoned that if ASD affects the direct subcortical inputs to the amygdala (Vuilleumier et al., 2003, 2004; Carretié et al., 2007; Rotshtein et al., 2007), ASD individuals should exhibit a reduced neural response to emotional 
expressions conveyed by LSFs. On the other hand, if ASD individuals present a reduced neural response to emotions mediated by HSFs, this can be interpreted exclusively as reflective of an effect to the cortical path.

\section{MATERIALS AND METHODS PARTICIPANTS}

Two groups were included in the experiment. The first group comprehended 13 high-functioning adults males with autistic spectrum disorder (ASD) recruited from the database of the Specialized Clinic for Pervasive Developmental Disorders of Rivière-des-Prairies Hospital. Diagnosis of autism was established with the Autism Diagnostic Interview-Revised (ADI-R; Lord et al., 1994) and validated by a standardized assessment with the Autism Diagnosis Observation Schedule (ADOS-G, module 3 or 4; Lord et al., 2000). All participants from the clinical group met the diagnostic criteria for autism or Asperger syndrome according to both instruments. The second group comprehended 15 matched male participants with typical development recruited from the same database.

Some of the participants were excluded from the overall analysis due to technical problems occurred during the acquisition phase and due to head-movement artifacts in the BOLD signal. Therefore, the overall analysis was run on two homogeneous groups of 10 individuals each. Participants from both groups completed one of the Wechsler Intelligence scales (WAIS-R,

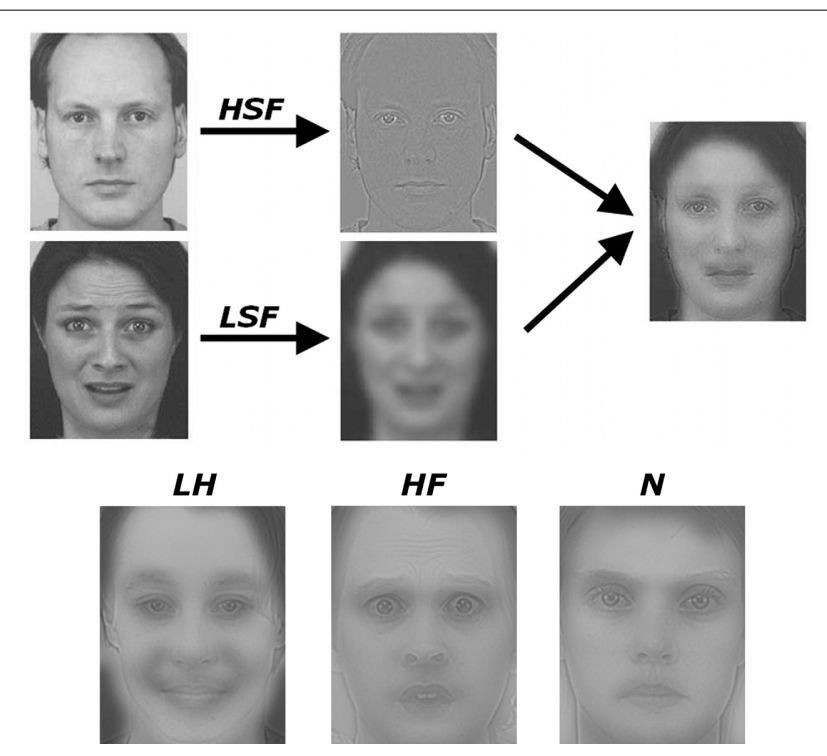

FIGURE 2 | Schematic representation of how the hybrid stimuli were created. Male and female faces were selected from a validated database and subjected to spatial frequency filtering. Hybrid faces were then created by overlaying a high-spatial frequency (HSF) face with a low-spatial frequency (LSF) face of opposite gender. Emotional expressions (in either the HSF or LSF face) were always overlaid with a neutral facial expression of opposite gender and opposite filtering. Three stimuli examples are also displayed on the lower part of the Figure. A HSF neutral face overlaid with a happy LSF face (condition LH), a HSF fearful face overlaid with a neutral LSF face (condition HF) and a control condition containing neutral faces in both HSF and LSF bands (N). Full details in the text.
WAIS-III) and the Edinburgh questionnaire (Oldfield, 1971). ASD and Control participants were group-wise matched according to their IQ [Manova on all IQ variables: Pillai's trace $=0.15$, $F_{(3,15)}=0.92$, n.s.], age and handedness. Table 1 summarizes the participants' demographic characteristics. Each participant gave informed consent to participate in the study and received monetary compensation. The study was formally approved by the ethics committee of Rivière-des-Prairies Hospital and the committee for ethics and research of Regroupement Neuroimagerie/Québec (CMER-RNQ). All participants were naïve to the purpose of the task.

\section{STIMULI}

Our experimental stimuli were hybrid images built by combining a face composed only by HSFs with a face composed only by LSFs of opposite gender, whose expression was also separately manipulated (see Figure 2 ). The detailed procedure was adapted from the one used by previous studies (Schyns and Oliva, 1999; Winston et al., 2003b) and can be summarized as follows. We took pictures of emotional (happy and fearful) and neutral facial expression, displayed from a frontal point-of-view, from the Karolinska Directed Emotional Faces database (A series). Validation studies on stimuli from this database (Goeleven et al., 2008) confirmed that happy and fearful expressions were matched for intensity and arousal, and both associated with higher recognition scores than neutral expressions. These images were desaturated, scaled to a size of about $5.30^{\circ}$ (horizontal) $\times 6.80^{\circ}$ (vertical) of visual angle and, subsequently, filtered in Fourier space, using a Butterworth filter to remove either high spatial frequencies (above $24 \mathrm{cycles} / \mathrm{face}[\mathrm{c} / \mathrm{fw}$ ], corresponding to about 4 cycles/degree of visual angle [c/deg]) or low spatial frequencies (below $6 \mathrm{c} / \mathrm{fw}$, corresponding to about $1 \mathrm{c} / \mathrm{deg}$ ). Hybrid stimuli were then created by overlapping one HSF face and one LSF face into a single stimulus (see Figure 2). The eyes and mouth position was matched between the LSF and HSF images in order to obtain a visual overlap yielding the percept of single face. Critically, faces in the LSF and HSF images were chosen so that they always had an opposite gender (one female, one male) and could display different emotional expressions. This manipulation led to the following five conditions of interest: high fearful stimuli, composed by a fearful HSF face and a neutral LSF face (HF); high happy stimuli,

Table 1 | Demographic and clinical characteristics of the sample.

\begin{tabular}{lcc}
\hline & $\begin{array}{c}\text { ASD participants } \\
(\boldsymbol{n}=\mathbf{1 0})\end{array}$ & $\begin{array}{c}\text { Neurotypical } \\
\text { controls }(\boldsymbol{n}=\mathbf{1 0})\end{array}$ \\
\hline Age (years) & $21.50[19.50,23.50]$ & $21.55[19.89,24.84]$ \\
Full scale IQ & $100.90[94.80,110.60]$ & $109.50[104.90,114.90]$ \\
Performance IQ & $98.70[92.90,104.80]$ & $103.00[99.33,109.70]$ \\
Verbal IQ & 102.50[96.20, 112.90] & $111.78[105.22,117.11]$ \\
Diagnosis & 6/10 Asperger syndrome & \\
& 4/10 High-functioning autism & \\
\end{tabular}

Average age and 10 values are displayed in average values with bootstrap estimated $95 \%$ confidence intervals. None of the displayed measures varied significantly across groups with $\alpha=0.05$ 
composed by a happy HSF face and a neutral LSF face (HH); low fearful stimuli, composed by a neutral HSF face and a fearful LSF face (LF); low happy stimuli, composed by a neutral HSF face and a fearful LSF face (LH); neutral stimuli (high-level control), composed by a neutral HSF face and a neutral LSF face $(N)$. For each of these conditions we built 32 different images, each of which was presented twice during the experimental session (32 images $\times 2$ repetitions $\times 5$ conditions $=320$ face stimuli). In half of these 32 pictures of each emotional condition, the emotional expression was conveyed by the female face, whereas in the remaining half the emotional expression was conveyed by a male face.

\section{EXPERIMENTAL SETUP}

The 320 face stimuli used in the study were presented to participants in an event-related fashion. On each experimental trial, one hybrid face was shown to the participant for $83 \mathrm{~ms}$ and followed by an inter-stimulus interval of variable duration (range 2500-12500 ms) in order to improve sensitivity of fMRI BOLD measurements. To encourage participants to keep their gaze on the center of the screen, a fixation cross was present during the inter-stimulus interval. The whole experiment was organized into four experimental sessions, each comprehending 80 trials (16 per condition) and lasting about $4 \mathrm{~min}$. Among these four sessions, two were associated with an active gender detection task: since participants were not aware that the hybrid stimuli were created by faces of opposite genders, they were requested to indicate, as fast as possible, its apparent gender by pressing one of two possible keys with either hand (e.g., left hand for male response, right for female, counterbalanced across participants). Previous studies using hybrid stimuli filtered at the same cut-offs found that, with such short stimulus presentations, participants rely with comparable likelihood on LSF and HSF information to make their gender judgments, as they report the gender of the LSF face on $\sim 50 \%$ of the trials (Schyns and Oliva, 1999; see also Winston et al., 2003b, in which the LSF face was chosen $60 \%$ of the trials). The two remaining sessions had no active task, and participants were simply requested to pay attention to each and every face. The order between passive and task-positive sessions was counterbalanced across subjects.

The stimuli were presented using E-Prime 1.0 (Psychology Software Tools, Inc.) and projected inside the scanner bore with a LCD projector on a screen subtending about $19^{\circ}$ (horizontal) $\times 14^{\circ}$ (vertical) of visual angle. Key-presses were recorded on an MRI-compatible bimanual response button box. Participants were instructed to press one of two possible keys, placed at each hand's reach, to indicate their responses.

\section{FACE FUNCTIONAL LOCALIZER}

Our study aimed at investigating the sensitivity to band-filtered face information in key areas of the core face processing system, particularly fusiform cortex and amygdala (Haxby et al., 2000; Gschwind et al., 2012). To this aim, we mapped the face processing network in both groups with an unbiased (not band-filtered) set of face stimuli. We therefore carried out an independent scanning session adapted from previous studies (Schwarzlose et al., 2005; Spiridon et al., 2006) and structured as follows. Participants were presented four blocks of gray-scale full-band face photographs alternating with four blocks of gray-scale house photographs. Photos were displayed centrally, and had a size of about $9.82^{\circ} \times 9.82^{\circ}$ ). Within each block, there were 18 face/house specimens each presented for $750 \mathrm{~ms}$ followed by an interstimulus interval of $500 \mathrm{~ms}$. Each block lasted of about $22 \mathrm{~s}$ each and was immediately followed by another. Whilst perceiving these images participants performed a 1-back task, in which they had to signal through key-press whether the picture in the current trial was identical to the one in the previous trial. The experiment was built so that a positive response from the participant was expected only in two trials in each block. The whole localizer session lasted about $3 \mathrm{~min}$ and always followed the four main experimental sessions.

\section{IMAGING PROCESSING \\ Data acquisition}

The study was conducted in the neurofunctional imagery unit at the research center of the geriatric institute of Montreal. A Siemens Trio 3-T whole-body scanner was used to acquire gradient-echo planar T2-weighted MRI images with blood oxygenation level dependent (BOLD) contrast. The scanning sequence was a trajectory-based reconstruction sequence with repetition time (TR) of $2160 \mathrm{~ms}$, echo time (TE) of $30 \mathrm{~ms}$, flip angle of $90^{\circ}$, in-plane resolution $64 \times 64,36$ slices, slice thickness of $3 \mathrm{~mm}$, and no gap between slices. A structural image was of each participant was also recorded with a T1-weighted MPRAGE sequence ( 176 slices, $T R=9.7 \mathrm{msec}, T E=4 \mathrm{~ms}$, flip angle $=12^{\circ}$, in-plane resolution $=256 \times 256,1 \times 1 \times 1 \mathrm{~mm}$ voxel size $)$.

\section{Preprocessing}

Statistical analysis was performed using the SPM software (http:// www.fil.ion.ucl.ac.uk/spm/). For each subject, all functional images were realigned, slice-time corrected to allow a whole volume to be treated as a single data point, normalized to a template based on 152 brains from the Montreal Neurological Institute $(\mathrm{MNI})$, resliced at a voxel size of $3 \times 3 \times 3 \mathrm{~mm}$, and then smoothed by convolution with a $8 \mathrm{~mm}$ full-width at halfmaximum (FWHM) Gaussian kernel.

\section{First-level analysis}

Data from each participant were analyzed using the General Linear Model (GLM) framework implemented in SPM. For the face localizer session, we modeled each of the two active conditions (faces, houses) with a boxcar function. For the main experimental sessions, the trial onsets from each condition of our design were modeled with a delta (stick) function. Critically, whereas in the two passive viewing sessions we modeled only the main five conditions of our design (HF, HH, LF, LH, N), in the gender discrimination task we also took into account participants' response on every trial (see Winston et al., 2003b). Thus, for each of the five main conditions, we modeled separately those trials in which participants made their gender judgments on the basis on visual cues conveyed by LSFs (e.g., HFL, HHL, LFL, LHL, NL), those trials in which participants judged gender based on HSFs (HFH, HHH, LFH, LHH, NH), and also those few trials in which responses were omitted (if any). Each regressor was convolved with a canonical hemodynamic response function as 
implemented in SPM. To account for movement-related variance, we included, for each session, six differential movement parameters $[\mathrm{x}, \mathrm{y}$, and $\mathrm{z}$ translations (in millimeters) and pitch, roll, and yaw rotations (radiants)] as covariates of no interest. Low-frequency signal drifts were filtered using a cutoff period of $128 \mathrm{~s}$.

\section{Second level analysis}

For the functional localizer, we calculated for each participant the contrast describing the differential activity Faces $>$ Houses. These contrasts were fed in a second-level independent sample $t$-test, under the assumption of unequal variance between the groups. This test allowed us to investigate both effects of Faces vs. Houses in Controls and ASD participants, as well as cross-over interaction effects.

For the main experiment, we considered for each subject 15 contrast images. 10 of them were computed from the gender discrimination task, and concerned activity associated with the five main conditions and the two possible responses (i.e., HFL, HFH, HHL, HHH, LFL, LFH, LHL, LHH, NL, NH). The remaining five concerned activity in the five conditions of interest (i.e., LF, LH, HF, HH, N) during the passive viewing sessions. These contrasts were fed into second-level flexible factorial design with "conditions" as a within-subject factor, "group" as betweensubject factor and "subject" as random factor, using a random effects analysis (Penny and Holmes, 2004). In modeling the variance components, we allowed each of these three factors to have unequal variance between their levels. Activations in these analyses were considered as significant if exceeding an extent threshold allowing $p<0.05$ correction for multiple comparison for the whole brain (corresponding to 59 and 63 consecutive voxels, for the localizer and main experiment respectively-Friston et al., 1993), with an underlying height threshold corresponding to $p<$ 0.001 uncorrected $\left[t_{(18)}>3.61\right.$ and $t_{(250)}>3.13$, for the localizer and main experiment].

\section{RESULTS \\ BEHAVIORAL RESULTS}

To obtain a measure of spatial frequency biases in face processing for different conditions in each group, we analyzed the rate at which participants selected the gender of the LSF face in the hybrid stimuli. In this measure, values greater than 0.5 reflect experimental conditions in which participants relied more on the LSF information to make gender judgments, whereas values smaller than 0.5 reflect conditions in which participants relied more on the HSF information.

We first analyzed the conditions in which an emotional expression was displayed through a $2 \times 2 \times 2$ Repeated measures ANOVA, with the FREQUENCY containing the emotional expression (HSF, LSF) and the VALENCE of this emotional expression (Fearful, Angry) as within-subject factors, plus participant GROUP (ASD individuals, Controls) as between-subjects factor. We found a significant main effect of FREQUENCY $\left[F_{(1,18)}=9.79, p<0.01\right]$, reflecting that overall participants relied more on LSF information [average 0.56, bootstrapestimated $95 \%$ confidence intervals of the average $(0.46,0.64)]$, rather than on HSF $[0.52(0.40,0.62)]$. However, this LSF-bias also depended on the valence of the emotion expression (see Figure 3). Thus, whereas the VALENCE main effect was not significant $\left[F_{(1,18)}=0.14\right]$, this factor interacted significantly with FREQUENCY $\left[F_{(1,18)}=18.48, p<0.001\right]$. Figure 2 shows that, in both groups, gender judgments were more LSF-biased when low frequencies conveyed happy expressions, as opposed to fearful $\left[L H>L F: t_{(19)}=2.39, p<0.05\right]$. Instead, judgments were more HSF-biased when high frequencies conveyed happy, as opposed to fearful, expressions $\left[H F>H H: t_{(19)}=2.96, p<0.01\right]$. The factor GROUP yielded no significant main effect nor interaction $\left[F s_{(1,18)}<1.00\right]$. Visual inspection of Figure 3 suggests that ASD individuals might be more LSF-biased than controls, although no significant effect of the factor GROUP was found. However, this initial analysis did not comprehend the high-level control condition in which neutral facial expressions were presented. We therefore also tested for putative group differences in LSF-rate, both when taking each of the five main conditions (thus including $\mathrm{N})$ separately, and when averaging them together. None of these tests led to a significant effect $\left[|t|_{(18)}\right.$ always $\left.<1.60\right]$.

Furthermore, for each condition, we computed the median time [in milliseconds (ms)] necessary to deliver a response (Response Times) and analyzed it in a similar fashion as above. In this analysis we also tested for any putative effect of the participants' choice. We therefore analyzed the emotional conditions in a $2 \times 2 \times 2 \times 2$ repeated measures ANOVA with FREQUENCY (HSF vs. LSF), VALENCE (Fear vs. Happy), and CHOICE (HSF vs. LSF gender) as within-subject factor and GROUP (Controls vs. ASD individuals) as between subject factor. We found a significant main effect of FREQUENCY $\left[F_{(1,18)}=4.96, p<0.05\right]$, reflecting faster responses when the emotional expression was conveyed by LSF $[825.50 \mathrm{~ms}(735.41,908.49)]$, as opposed to HSF $[870.51(769.19,944.95)]$. No other main/interaction effect, including those associated with the factor CHOICE, was significant $\left[F s_{(1,18)}<4.27, p s>0.05\right]$.

\section{NEURAL RESPONSES}

Face Localizer. Data from the Face localizer are displayed in Table 2 and Figure 4. We tested, in each group, whether there were significant differences in neural activity between the Face and House categories. The contrast Faces $>$ Houses confirmed, in both neurotypical (Figure 4, red clusters) and ASD individuals (green clusters), an involvement of the amygdala and of the posterior portion of the superior temporal sulcus in the two hemispheres. Controls also exhibited activation the medial orbitofrontal cortex. No fusiform activation was found in either group at the whole-brain threshold. We therefore performed additional region-of-interest analyses restricted to those voxels that were part of these fusiform gyrus as described by predefined anatomical masks (AAL database-Tzourio-Mazoyer et al., 2002). In Controls, we found bilateral activation of the fusiform gyrus, $\sim 45 \mathrm{~mm}$ posteriorly from the anterior commissure, over and around the region usually identified as FFA. No suprathreshold activation was found in ASD participants, although at a less stringent height threshold (corresponding to $p<0.005$ uncorrected) activation was found around the same FFA coordinates as defined in the Control group for both the right (12 consecutive voxels centered at the coordinates $x=42, y=-48$, 

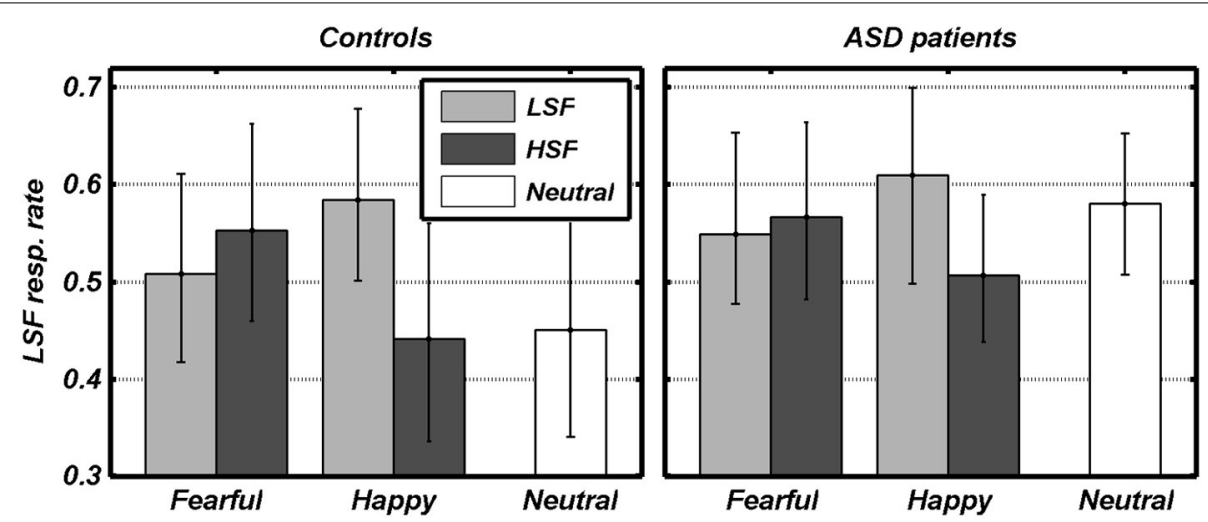

FIGURE 3 | Behavioral data. The rate of gender judgments made according to the LSF information in each condition is plotted against the valence of facial expressions. Dark and light gray bars refer to the different spatial frequencies conveying emotional expression. White bars refer to control trials with no emotional expression. Data from each group are displayed in separate subplots. Error bars refer to bootstrap-based $95 \%$ confidence intervals of the mean. $z=-21$ ) and left hemisphere (three consecutive voxels centered at $x=-39, y=-48, z=-21)$. The opposite contrast (Houses $>$ Faces) implicated large portions of the parahippocampal gyrus, extending to the calcarine cortex and the medial portion of the middle occipital gyrus, in both groups similarly. When testing for the interaction between the grouping factor and the stimuli employed (Faces vs. Houses), we found no suprathreshold effects.

In sum, data from this localizer session successfully identified neural structures most sensitive to face stimuli, indicating the recruitment of similar portions of the fusiform cortex and amygdala in each group (although the evidence of FFA activity in ASD individuals was obtained with a more liberal threshold).

As the functional localizer aimed at mapping in our population those portions in fusiform cortex and amygdala that were most sensitive to full-band face stimuli in each group, we then used the results of the localizer session to create a mask which could serve as region of interest in all subsequent analyses. This mask was built following anatomical and functional criteria, as it included voxels which (1) were part of either the fusiform gyrus or the amygdala according to predefined anatomical masks (AAL database) and (2) exhibited significant $\left[t_{(18)}>1.73, p<0.05\right.$ uncorrected] increase of neutral activity for faces (as opposed to houses $)$ in each group [conjunction $\left((\text { Faces }>\text { Houses })_{\text {Controls }} \cap\right.$ (Faces $>$ Houses $\left.\left.)_{\mathrm{ASD}}\right)\right]$. The resulting mask, which was smoothed ( $8 \mathrm{~mm}$ FWHM Gaussian kernel) and subsequently re-binarized to minimize spatial inhomogeneities, encompasses that part of the fusiform-amygdala face network that is common to both groups.

\section{Effects of LSF emotional expressions}

We focused on that portion of the data in which Controls carried out the gender discrimination task and tested for increases of neural activity associated with LSF expressions, relative to neutral stimuli $[(L F L+L F H+L H L+L H H) / 2-(N L+N L)$, hereafter $L S F-N]$. When correcting for multiple comparisons across the whole brain we found no suprathreshold activation. However, when applying small-volume correction on those portions of the fusiform gyrus and amygdala identified in the localizer (see above), we found a significant increase of neural activity in the right fusiform cortex (see Table 3 and Figure 5A, red blob). This right fusiform activation was close, not only to the location previously identified by Winston et al. (2003b) in the same contrast (distance between the local maxima from the two studies $\sim 11 \mathrm{~mm}$ ), but also to the right FFA cluster isolated in the same group during the face localizer and displayed in Figure 4 (local maxima distance $\sim 15 \mathrm{~mm}$ ). No suprathreshold effect was found in the amygdala (similar to Winston et al., 2003b, but see Vuilleumier et al., 2003 who used simple band pass filtered stimuli).

One of the key questions of the present study was to assess whether this increase of neural activity in FFA for LSFs (as found in Controls) was absent or preserved in ASD individuals. We therefore examined the sessions in which ASD participants performed the gender discrimination task and tested for the same contrast $L S F-N$ : this revealed an activation of the left FFA, in a location very symmetrical to that identified in Controls (see Figure 5A, green blob-local maxima distance between this cluster and the left FFA cluster identified in the same group $\sim 6 \mathrm{~mm}$ ). No effect was found in the right fusiform gyrus or in the amygdala even at the most liberal thresholds.

We further explored putative group differences in the neural response to LSF emotional expressions by testing the interaction between the contrast $L S F-N$ and the grouping factor. In particular, we tested for regions in which the differential activity between LSF and neutral expressions in Controls was not only larger than 0 (as already tested above), but also larger than the same differential activity in ASD [i.e., $(L S F-N)_{\text {Controls }}-$ $\left.(L S F-N)_{\mathrm{ASD}}\right]$. However, as this test also isolates regions with no difference between LSF and neutral expressions in Controls, but with reduced activity for LSF expressions (as opposed to $N$ ) in ASD individuals, we excluded from our search those regions that were implicated ( $p<0.05$ uncorrected) in the contrast $N-L S F$ in ASD (exclusive masking). This test revealed no differential effect, neither when correcting for multiple comparisons for the whole brain, nor when focusing on the face-sensitive portions of fusiform gyrus/amygdala. With a similar logic, we tested for 


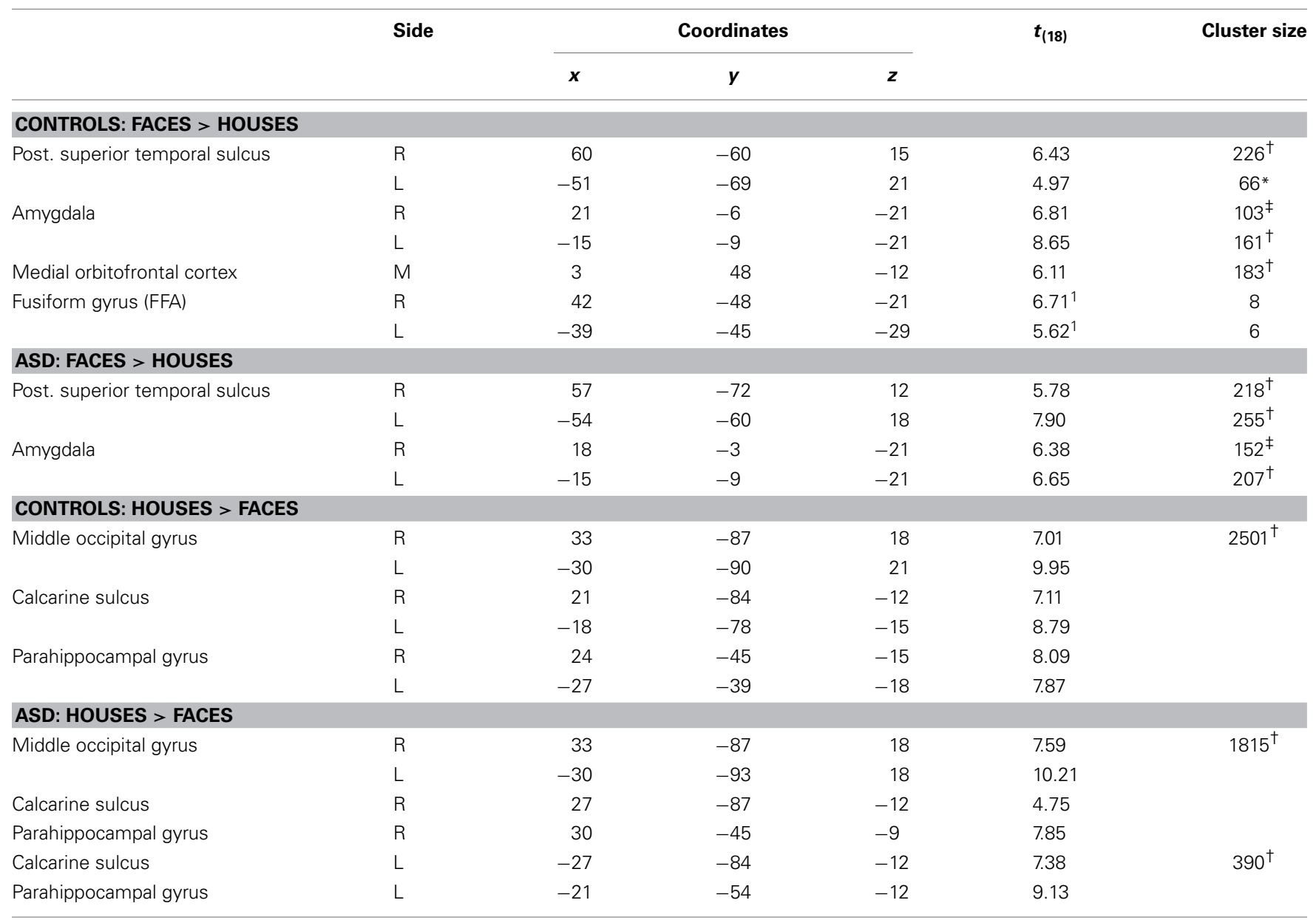

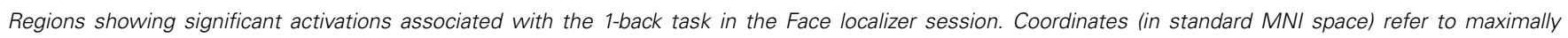

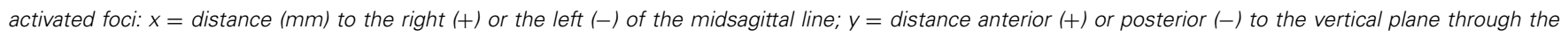

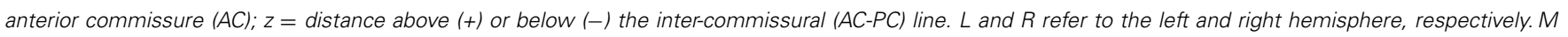
refers to medial clusters.

${ }^{t} p<0.001 ;{ }^{\ddagger} p<0.01 ;{ }^{*} p<0.05$ corrected at the cluster level for the whole brain (underlying height threshold: $p<0.001$, uncorrected).

${ }^{1} p<0.05$ corrected at the voxel level for the fusiform gyrus bilaterally.

regions in which the differential activity between LSF and neutral expressions was larger in ASD individuals than in Controls [i.e., $(L S F-N)_{\mathrm{ASD}}-(L S F-N)_{\text {Controls }}$ ]. Also this test led to no suprathreshold effects, including for the fusiform gyrus/amygdala at liberal thresholds.

In sum, not only we found reliable evidence in the neurotypical brain for a role of LSF inputs conveying emotional expression information to FFA (Winston et al., 2003b), but we also found equivalent (although contralateral) effects in ASD, suggesting that these LSF inputs are preserved in these participants. Furthermore, direct comparison of the effects identified in each group failed to reveal any significant difference.

\section{Effects of HSF emotional expressions}

We next tested for regions exhibiting suprathreshold activity when emotional face expressions were conveyed by HSFs. We first computed, in Controls, the contrast $[(H F L+H F H+$
$H H L+H H H) / 2-(N L+N L)$, hereafter $H S F-N]$ which revealed enhanced bilateral activity in the fusiform gyrus, over and around FFA, as well as in the Amygdala (see Table 3 and Figure 5B, red blobs). Critically, the fusiform clusters were proximal to the FFA clusters delineated with the functional localizer in the same group (Figure 4) and to the clusters identified by the main effect of LSF expressions (Figure 5A, red blobs). No effect was found for the contrast $H S F-N$ when ASD participants carried the discrimination task.

We then tested directly whether the differential activity observed in Controls was larger, not only than 0 , but also of its homologous in ASD individuals via an interaction test $\left[(H S F-N)_{\text {Controls }}-(H S F-N)_{\mathrm{ASD}}\right.$, excluding voxels sensitive to $\left.(N-H S F)_{\mathrm{ASD}}\right]$. We found no suprathreshold activity, neither when correcting for the whole brain, nor when applying a small volume correction. It should be mentioned, however, that under an uncorrected extent threshold (underlying 
Table 3 | fMRI analysis: effects of HSF and LSF emotional cues.

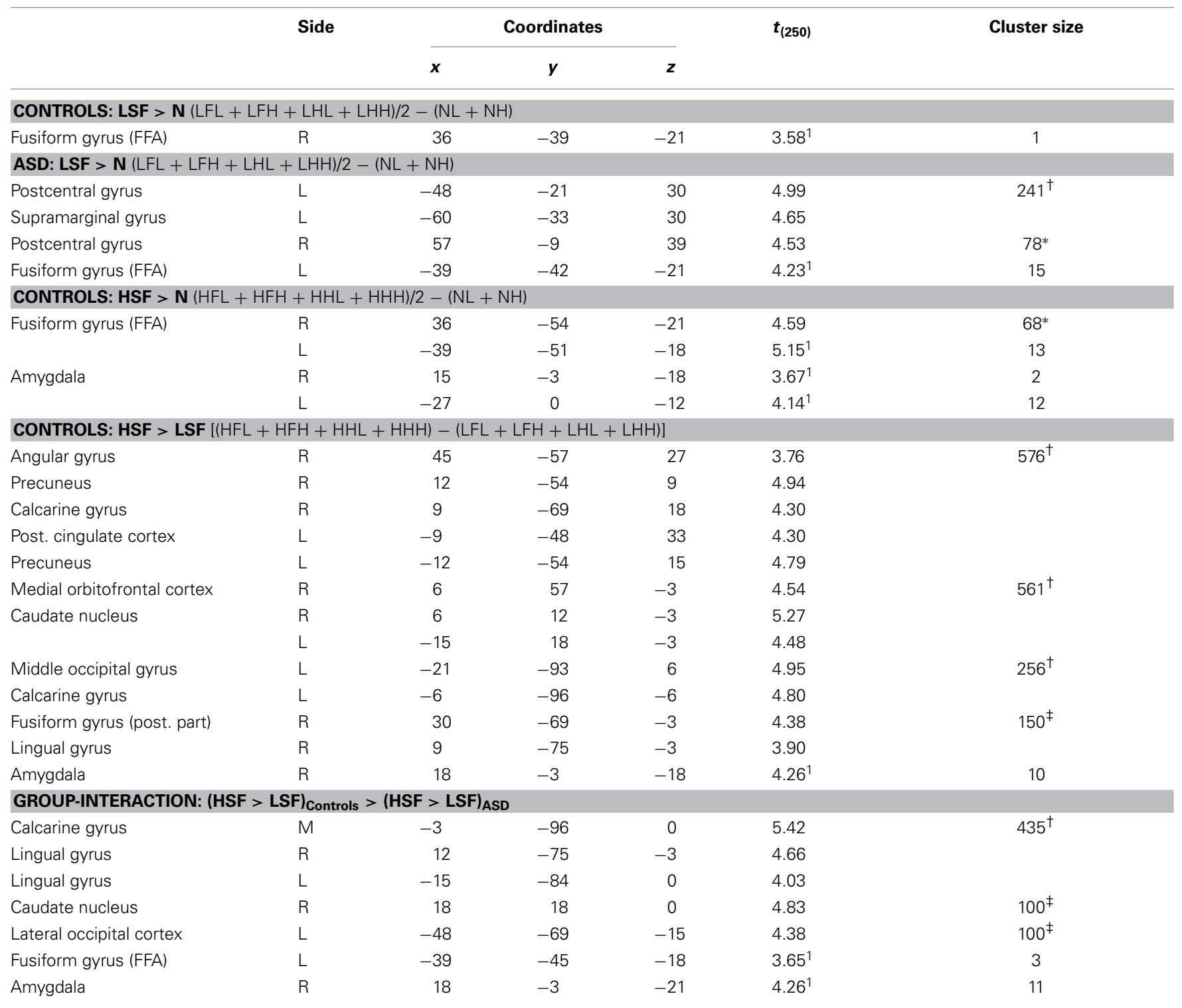

Regions showing significant activation associated with the discrimination task.

${ }^{t} p<0.001 ;{ }^{*} p<0.01 ;{ }^{*} p<0.05$ corrected at the cluster level for the whole brain (underlying height threshold: $p<0.001$, uncorrected).

${ }^{1} p<0.05$ corrected at the voxel level for FFA and amygdala bilaterally as described by the localizer data.

height threshold of $p<0.001$ ), we found five consecutive voxels on right FFA [local maxima: $x=33, y=-51, z=-21$, $\left.t_{(162)}=3.57\right]$, proximal to the cluster previously implicated when testing effects of HSF expressions (local maxima distance $<5 \mathrm{~mm})$. No region exhibited HSF increases of activity specific for ASD individuals $\left[(H S F-N)_{\mathrm{ASD}}-(H S F-N)_{\text {Controls }}\right]$, neither when correcting for the whole brain, nor when inspecting the fusiform gyrus and the amygdala with more liberal approaches.

In sum, the analysis of HSF effects during the discrimination task revealed significant increases of neural activity in FFA and amygdala to emotional expressions in the Controls exclusively. For the right FFA, such increase was not only larger than
0 , but also larger than the homologous (non-significant) effect measured in ASD individuals.

\section{Direct comparisons between LSF and HSF expressions}

We also compared differential responses to LSF or HSF expressions, not against the control neutral condition, but against each other. Unlike the analysis conducted insofar-which identified regions sensitive to one frequency band, irrespective of their sensitivity also to the other bands-these direct comparisons now probed for any region that would code preferentially for emotional information conveyed by specific frequencies.

When testing for differential responses to LSF expressions [contrast $(L F L+L F H+L H L+L H H)-(H F L+H F H+$ 


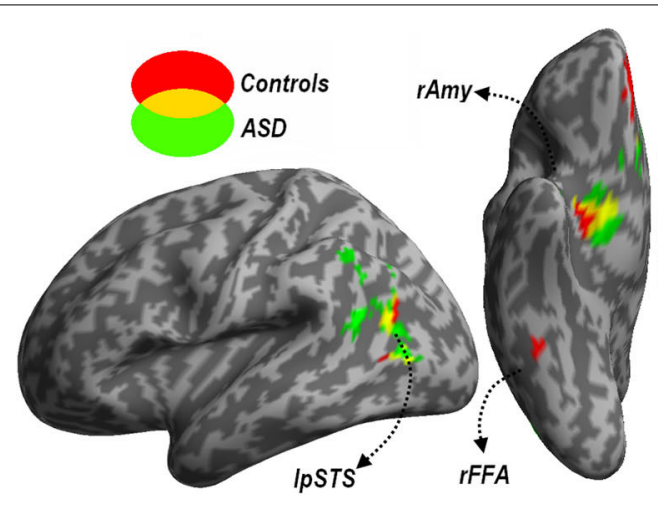

FIGURE 4 | Localizer session. Whole-brain maps showing significant increase of neural activity associated with the perception of Faces > Houses from the localizer session. Data from neurotypical participants are displayed in red, whereas data from ASD individuals are displayed in green (height threshold $p<0.001$ ). Overlaps between red and green regions are displayed in yellow. Additional overlaps were seen in the fusiform cortex at lower threshold only (see main text). Activations are

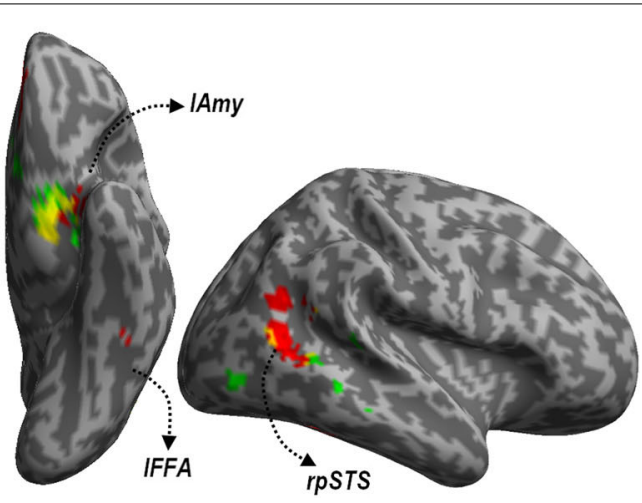

overlaid on an inflated brain surface. Three views are depicted: lateral view of left and right hemispheres (left and right side of the figure, respectively) and ventral view (center of the figure). Note that in the ventral view the right hemisphere is left to the left hemisphere. FFA, fusiform face area; Amy, amygdala; pSTS, posterior portion of the superior temporal sulcus; mOFC, medial orbitofrontal cortex; $r$ and I, right and left hemisphere respectively.
$H H L+H H L)$, hereafter $L S F-H S F]$, we found no suprathreshold effect in neither in Controls, nor in ASD individuals. We then tested the converse contrast HSF - LSF, which probed for any region processing emotional facial expressions from HSF cues preferentially to LSF cues. For Controls, this contrast elicited large activations within the ventral occipital cortex, including the posterior portions of the fusiform gyrus. Further activations were found in the right angular gyrus, the precuneus/posterior cingulate cortex, the ventral striatum bilaterally, the medial orbitofrontal cortex and, when applying small volume correction, the right amygdala (see Table 3 and Figure 6, red blobs). No effects (even at the most liberal thresholds) were observed when the same contrast was run on the ASD group.

In sum, these data confirm in Controls the recruitment of a widespread network processing emotional face expression from HSFs preferentially to LSFs. Such network was not reported in ASD individuals, not even at the most liberal thresholds. It is therefore possible that the same regions processing preferentially HSF in Controls might exhibit different sensitivity to spatial frequency emotional cues in ASD. We formally tested this via a cross-over interaction contrast $(H S F-L S F)_{\text {Controls }}-(H S F-$ $L S F)_{\mathrm{ASD}}$, comparing the differential sensitivity between HSF and LSF emotional cues across groups. As fully described in Figure 6 (yellow blobs) and Table 3, this analysis confirmed the role played by the lingual gyrus, the ventral striatum and the right amygdala. Furthermore, this analysis also implicated the left lateral occipital cortex and left FFA, thus confirming how this region seems more sensitive to HSF expressions in Controls and, concurrently, to LSF expressions in ASD individuals (see also Figure 5).

\section{Effects of the reported gender and of emotional valence}

All analyses conducted insofar were run regardless of the behavioral performance and of the emotional valence. Figure 7 illustrates the activity parameters extracted from those FFA and Amygdalar voxels identified by the contrasts $L S F-N$ and $H S F-N$ (Figure 5). Visual inspection of these data suggests how in some cases the differential activity between emotional and neutral expressions described above might be biased by the task demands. In particular, the amygdala exhibited, in Controls, a differential increase in activity for HSF expressions; however, further in-depth analyses on the extracted parameters revealed a general marginal preference for all trials in which HSF were "preferred" for the gender discrimination [choose HSF vs. choose LSF: right Amygdala $t_{(9)}=2.04, p=0.072$; left Amygdala $t_{(9)}=2.05, p=$ $0.071]$. Instead, amygdala activity seemed unaffected by the kind of emotion displayed by HSFs [fearful vs. happy: right Amygdala $t_{(9)}=0.87$, n.s.; left Amygdala $t_{(9)}=-0.13$, n.s.]. Keep in mind that the contrast $H S F-N$, implicating the amygdala in our earlier analyses (Figure 5B), was calculated by weighting equally the two possible gender choices.

On the other hand, we found that FFA activity to HSF (in Controls) and to LSF (in Controls for the right hemisphere, and ASD individuals in the left hemisphere) was globally unaffected by participants' behavior or by emotional valence $\left[|t|_{(9)}\right.$ always <1.60]. Visual inspection of Figure 7D, suggests that, in ASD individuals, the processing of LSF happy expressions might elicit larger activity in left FFA for those trials in which a HSF gender was chosen as opposed to a LSF gender. This was confirmed by an ad-hoc comparison [LHH vs. LHL, $t_{(9)}=2.76, p<0.05$ ].

Finally, we extended the results obtained in FFA and amygdala to the whole brain, by assessing for each group the putative effects of the behavioral choice (HSF vs. LSF gender) and of emotional valence (fearful vs. happy). However, this analysis led, in its most relevant contrasts, to no suprathreshold activity. Specifically, neither Controls nor ASD individuals exhibited any suprathreshold effect associated with emotional valence, neither when testing the overall main effect [contrast $(L F L+L F H+H F L+$ $H F H)-(L H L+L H H+H H L+H H H)$ and inverse], nor when focusing only on those trials in which emotions were conveyed by specific frequency bands [contrasts $(L F L+L F H)-$ $(L H L+L H H),(H F L+L F H)-(H H L+H H H)$, and inverses]. No suprathreshold effect was found when testing whether there 


\section{A Effects of Low spatial frequencies}
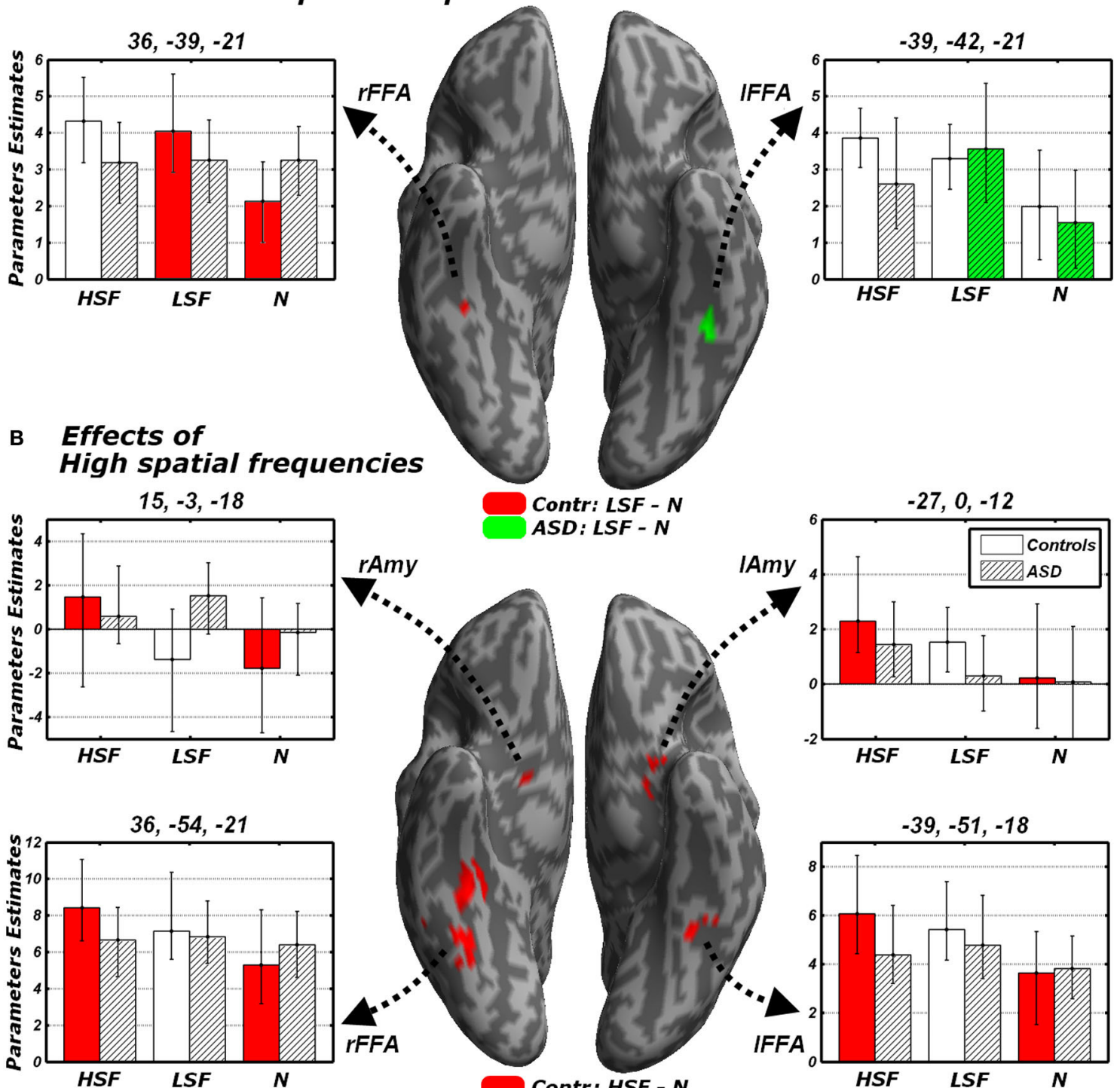

$A S D$ : $L S F-N$

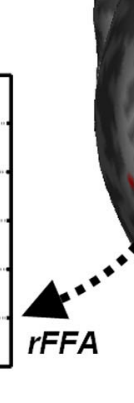

FIGURE 5 | Discrimination task: independent effects of emotional information in either LSF (A) or HSF (B) relative to neutral control stimuli. Whole-brain maps showing significant increase of neural activity associated with $(\mathbf{A})$ the contrast $L S F-N$ or $(\mathbf{B})$ the contrast $H S F-N$ in Controls (red blobs) and ASD individuals (green blobs). Activations are overlaid on an inflated brain surface. Suprathreshold activations were found in fusiform gyrus (over and around FFA) and the amygdala. Parameters extracted from each cluster are displayed bootstrap-based 95\% confidence intervals of the mean. Empty bars refer to data from Controls whereas striped bars refer to data from ASD individuals. Color codes on the bar graphs refer to conditions in the statistical test used to identify each region. FFA, fusiform face area; Amy, amygdala; $r$ and I, right and left hemisphere respectively. were regions affected by participants' choice [contrast ( $L F L+$ $L H L+H F L+H H L)-(L F H+L H H+H F H+H H H)$ and vice versa]. As in the case of the amygdala (Figures 7A,B), we tested putative effects of choice within those frequency bands conveying emotional information (choose HSF $>$ choose LSF only for HSF emotional expressions, or choose LSF > choose HSF only for LSF expressions), but no significant effect was found in any of the groups. No suprathreshold effect was found associated with the interaction between the frequency conveying an emotional expression and the frequency promoting the gender choice, specifically when searching for regions with higher activity in trials in which the face with an emotional expression was chosen rather than neglected [contrast $(L F L+L H L+H F H+$ $H H H)-(L F H+L H H+H F H+H H H)]$. Finally, in keeping with our behavioral finding that participants' response was affected by the emotional content of happy expressions only, we excluded from the interaction contrast those trials displaying fearful faces [contrast $(L H L+H H H)-(L H H+$ $H H H)]$, but even in this case we found no suprathreshold activity. 


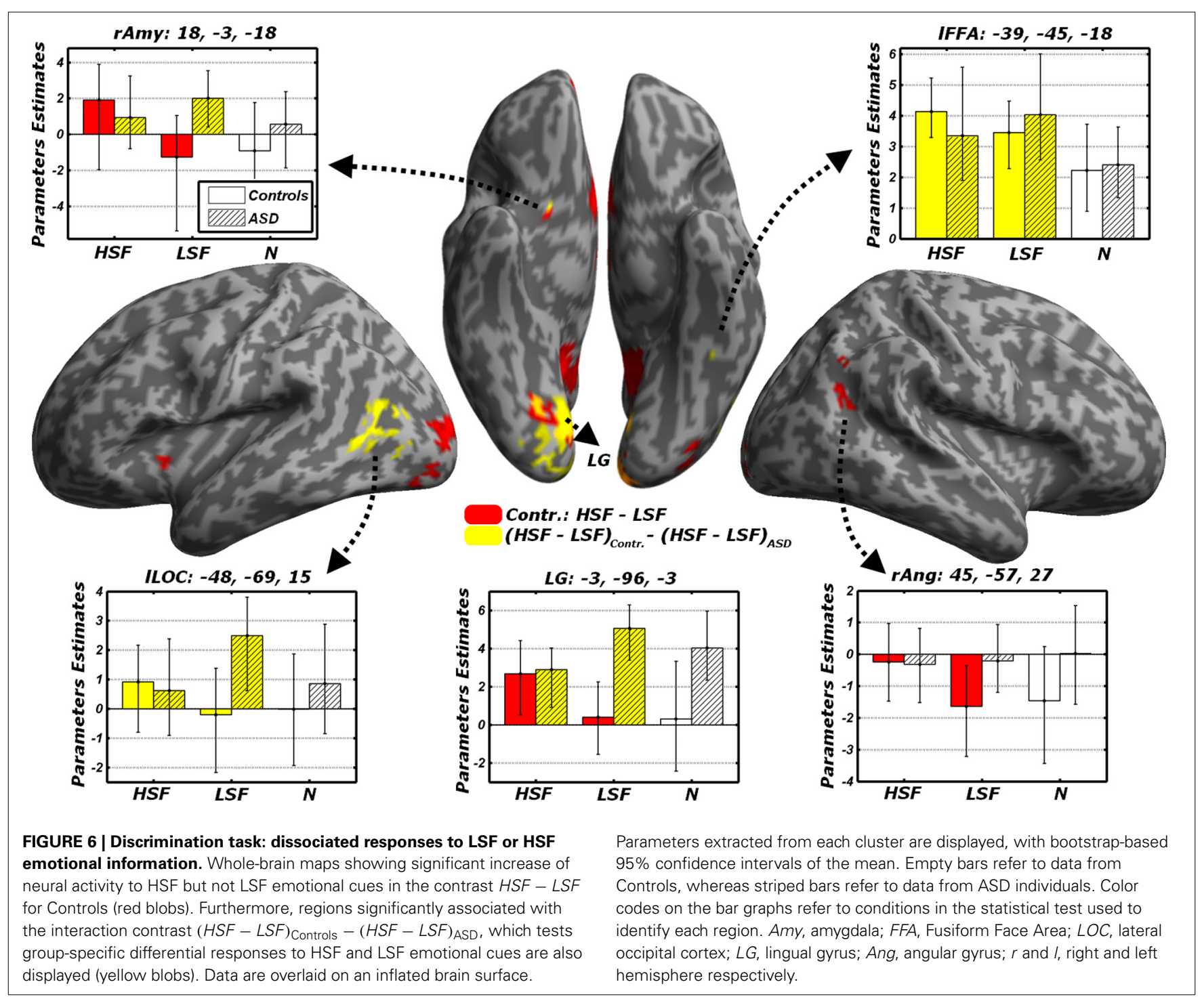

\section{Passive viewing trials}

Finally, all effects associated with the passive viewing trials are displayed in Table 4 and can be summarized as follows. No region was uniquely recruited by the perception of LSF emotional expressions as opposed to neutral stimuli $(L F+L H) / 2-$ $N$, neither in Controls nor in ASD individuals. Instead, ASD individuals (but not Controls) exhibited increased activity in the right fusiform gyrus for HSF expressions [contrast $(H F+$ $H H) / 2-N]$, in proximity to the region identified through the same contrast in Controls when testing the gender discrimination sessions (see Figure 8A, green blob). We then inspected any effect of emotional valence, both as a global main effect [contrast $(L F+H F)-(L H+H H)$ and inverse] and by analyzing separately each frequency band. Controls exhibited only enhanced activity of the most anterior portion of the left fusiform gyrus, extending to the parahippocampal cortex, for HSF happy (relative to HSF fearful) expressions (see Figure 8A, red blobs).

On the other hand, ASD individuals exhibited increased activity in the left middle-anterior insula for fearful (as opposed to happy) expressions, regardless of the spatial frequency. Such effect was not observed when repeating the analysis separately for each frequency band. Furthermore, in ASD individuals, LSF happy expressions triggered (compared to LSF fearful expressions) enhanced activity in the most ventro-lateral part of the right amygdala (Figure 8B, green blob). Finally, in ASD individuals, the contrast $(H H-H F)$ elicited significant differential activation in the temporo-parietal junction (bilaterally), the posterior cingulate cortex and the left superior frontal sulcus.

In sum, in sharp contrast with the case of the Gender Discrimination task, during the passive viewing sessions ASD individuals exhibited increased neural responses in portions of the core face network for emotional facial expressions, including those conveyed by HSFs.

\section{DISCUSSION}

We tested for the independent contribution of HSF or LSF visual inputs to brain regions critical for face processing, by engaging individuals with ASD and matched neurotypical Controls in a 


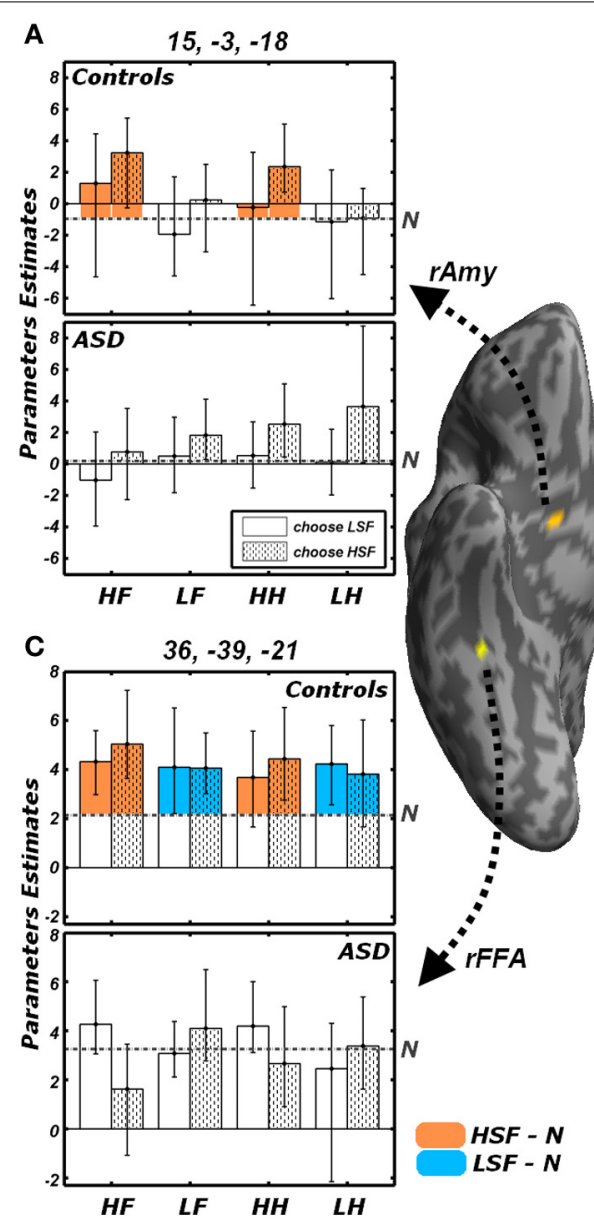

FIGURE 7 | Effects of the reported gender and of emotional valence. Average parameters extracted from representative voxels of (A) right Amygdala, (B) left Amygdala, (C) right FFA and (D) left FFA. The left and right FFA voxels were chosen as exhibiting significant conjoint activity for the contrasts $L S F-N$ (as shown in Figure 5A) and $H S F-N$ (Figure 5B). Amygdalar voxels were those composing the clusters depicted in

Figure 5B. The four regions are displayed in yellow on a ventral portion of an inflated human brain. For each of these four regions, average parameters estimates are displayed with bootstrap-based $95 \%$ confidence intervals of the mean. Data from different groups are displayed in separate subplots. Empty bars refer to trials in which participants choose the gender depicted

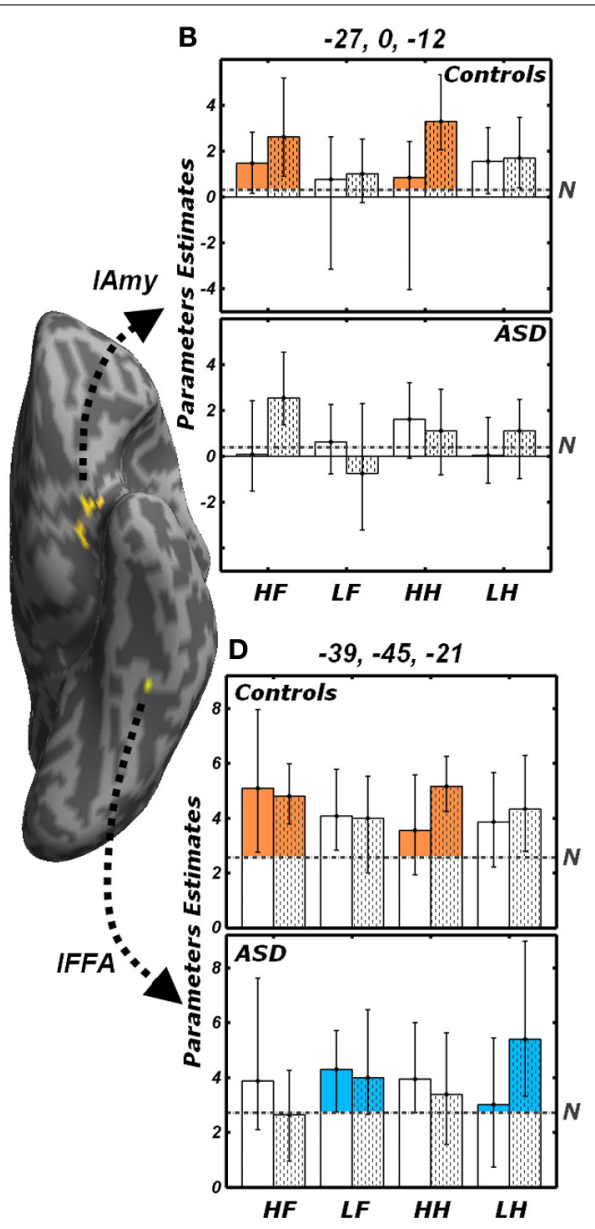

by the LSF, whereas dotted bars refer to trials in which the HSF gender was chosen. The average activity associated with the neutral condition is displayed as a gray horizontal dash-dotted line. The portions of the bars which exceed the activity of the neutral condition are colored according to the functional test with which the regions were defined. Regions isolated through the contrast $L S F-N$ (Figure 5A) display the bars associated with LSF conditions colored in blue; instead regions isolated through the contrast HSF - N (Figure 5B) display the bars colored in orange. HF, HSF fearful expression; LF, LSF fearful expression; HH, HSF happy expression; LH, LSF happy expression; N, Neutral expression; Amy, amygdala; FFA, Fusiform Face Area; $r$ and I, right and left hemisphere respectively. gender discrimination task with hybrid face stimuli. We found that, compared to Controls, individuals with ASD exhibited a reduced sensitivity to emotional information conveyed by the cortical HSF pathway, but were as sensitive as Controls to information conveyed by the LSF pathway. This was observed both in the portion of fusiform gyrus sensitive to face stimuli (FFA), when measuring the neural responses to emotional expressions in either frequency against control neutral faces (Figure 5), and in both the ventral occipital cortex and the amygdala when testing HSFs against LSFs (Figure 6, red blobs). FFA, the ventral occipital cortex and the amygdala were also showed a significant interaction reflecting that the increased activity for HSF expressions observed in Controls was reliably larger than this effect in ASD individuals (Figure 6, yellow blobs). Furthermore, both FFA and amygdala responses to emotional cues seem independent from the emotional valence, whereas they were modulated by the participants' choices in the gender task—at least for the amygdala (Figure 7). Critically, these data cannot be interpreted as ASD being characterized by a generalized insensitivity to HSF cues conveyed by the cortical pathway per se, because posterior visual cortical regions responded to HSF emotional information in ASD individuals during the passive viewing sessions (Figure 8). Instead, the data suggest decreased sensitivity to HSF information when processing global facial features, such as during active gender discrimination.

\section{LOW- AND HIGH-FREOUENCY PROCESSING IN ASD}

The hybrid nature of our stimuli, and LSF and HSF cut-offs adopted in keeping with our previous studies $(<6 \mathrm{c} / \mathrm{fw}$ and $>24$, 
Table 4 | fMRI analysis: regions showing significant activation associated with the passive viewing sessions.

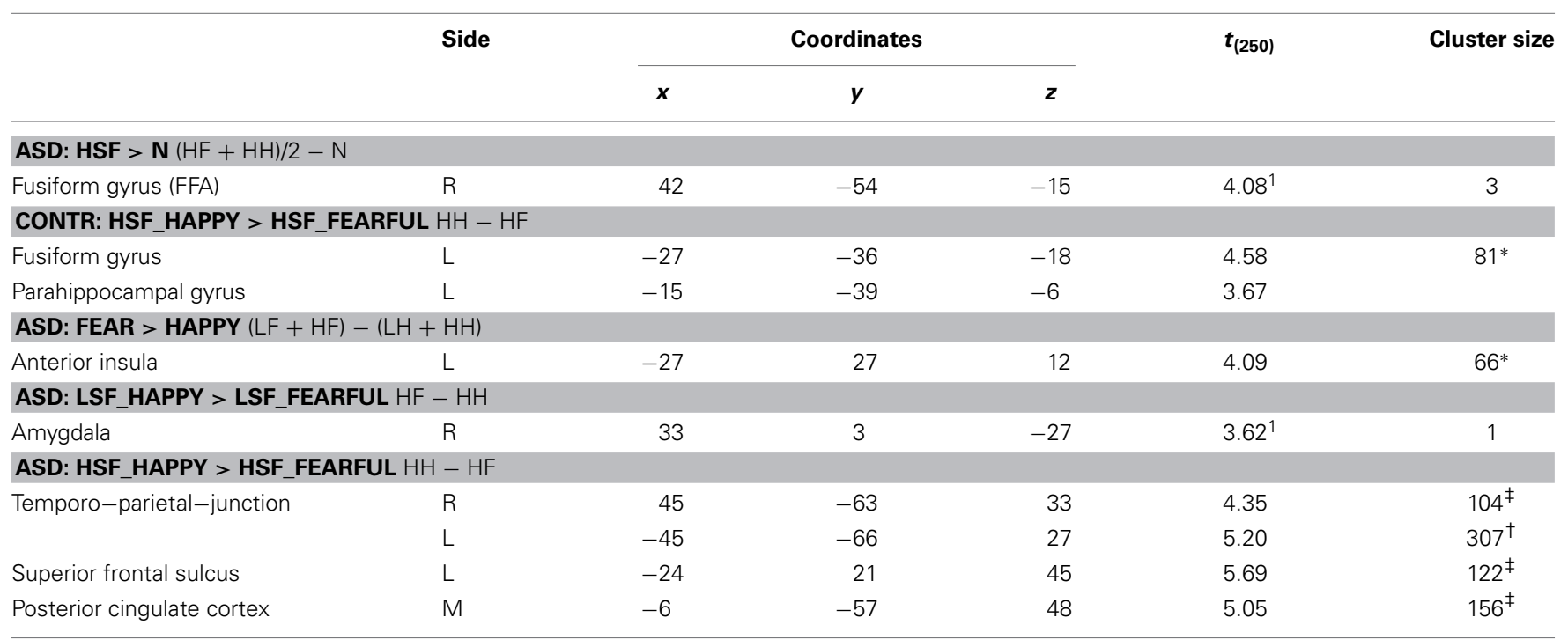

${ }^{+} p<0.001 ;{ }^{\ddagger} p<0.01 ;{ }^{*} p<0.05$ corrected at the cluster level for the whole brain (underlying height threshold: $p<0.001$, uncorrected).

${ }^{1} p<0.05$ corrected at the voxel level for FFA and amygdala bilaterally as described by the localizer data.

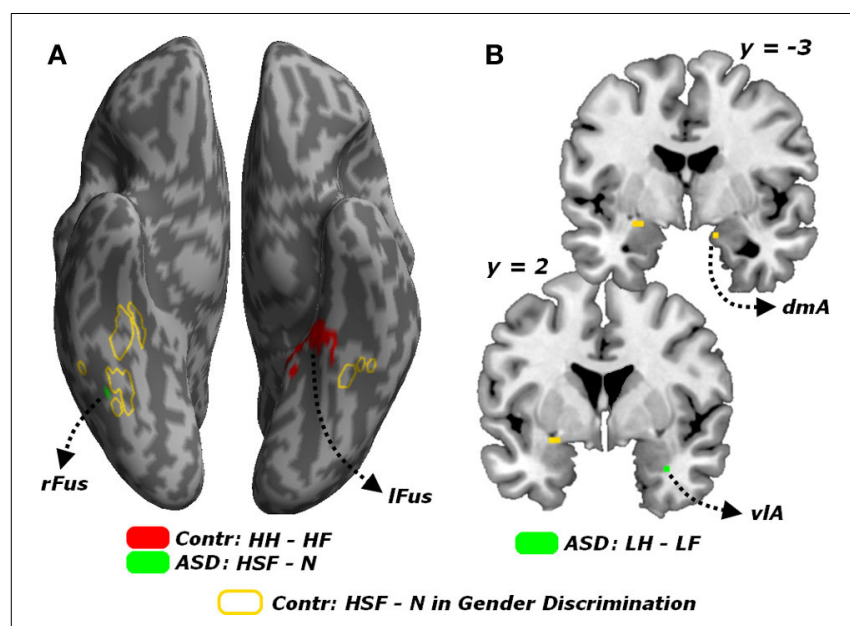

FIGURE 8 | Passive viewing sessions. (A) Whole-brain maps showing significant increase of neural activity associated with the contrast $\mathrm{HH}-\mathrm{HF}$ in Controls (red blob) the contrast HSF - N in ASD individuals (green blob). Activations are overlaid on an inflated brain surface. (B) Coronal sections $(y=-3,2)$ displaying the increase of neural activity for the contrast $L H-L F$ in ASD individuals (green blobs) in the right amygdala. For both $(\mathbf{A}, \mathbf{B})$ portions of fusiform and amygdalar cortex implicated in the contrast $H S F-N$ in earlier analysis on the gender discrimination sessions are displayed in yellow. Fus, Fusiform Gyrus; $r$ and I, right and left hemisphere respectively. $d m A$ and $v / A$, dorsomedial and ventrolateral portions of the amygdala.

c/fw respectively_-Vuilleumier et al., 2003; Winston et al., 2003b; Pourtois et al., 2005) served two main purposes: first, they allowed co-occurrent, and yet dissociable, recruitment of parvocellular and magnocellular pathways; second they insured that spatial frequency information conveyed by each pathway provided coarse (LSF) and fine-grain (HSF) facial cues that were equally distant from optimality. With this set of stimuli, we found no behavioral difference between ASD individuals and Controls. Group differences were observed only when measuring neural responses, specifically for fine-grained information that is uniquely conveyed by the cortical pathway. Earlier studies using gratings stimuli of LSFs or HSFs found comparable contrast thresholds in ASD individuals and Controls (Bertone et al., 2005; De Jonge et al., 2007; but see Davis et al., 2006, for differences in HSF), but nevertheless documented atypical neural responses in ASD (for LSF, Boeschoten et al., 2007b; Vlamings et al., 2010; for HSF Boeschoten et al., 2007b; Milne et al., 2009).

Faces are much more complex stimuli as they are processed through the integration of co-occurrent HSF and LSF information arising from each pathway. Notably, earlier studies using simple band-pass filtered or hybrids faces often reported that ASD individuals might be more biased in favor of HSF than LSF (Deruelle et al., 2004, 2008), and exhibit atypical neural responses to LSF faces (Vlamings et al., 2010). It should be mentioned, however, that these studies differed from ours in many aspects, such as the recruitment of children (see Rondan and Deruelle, 2004, for a lack of effects in adults), the task employed (see Deruelle et al., 2008, who reported no HSF biases in gender discrimination task) and, critically, the use of a more liberal LSF cutoff $(<12 \mathrm{c} / \mathrm{fw})$. Indeed, psychophysical investigations in neurotypical individuals have consistently described that face information is optimally processed from intermediate frequency bands (between 8-16 c/fw - Costen et al., 1994, 1996; Gold et al., 1999; Näsänen, 1999; Parker and Costen, 1999; Boutet et al., 2003; Collin et al., 2006; Watier et al., 2010). In this perspective, previous studies should not be interpreted as showing atypical processing of LSFs per se, but of those intermediate frequencies optimal for face processing. Consistently with this conjecture, a study employing face stimuli filtered under a more stringent LSF cutoff $(<5 \mathrm{c} / \mathrm{fw}$ - thus, outside the range $8-16 \mathrm{c} / \mathrm{fw}$ ) reported no difference in neural activity 
between ASD children and matched Controls (Boeschoten et al., 2007a).

\section{GENDER DISCRIMINATION IN ASD}

The gender discrimination task employed here served the purposes of our study in three critical aspects. First, this task chiefly requires the inspection of faces from a global point of view, as shown by decreased performance when the face stimuli are inverted, scrambled, or when the upper and lower halves are misaligned (Zhao and Hayward, 2010). Second, the gender of hybrid stimuli can be discriminated by relying on either LSF or HSF bands (equally from both bands in Schyns and Oliva, 1999; slightly LSF-biased in Winston et al., 2003b). Third, the discrimination is influenced by the (taskirrelevant) emotional expressions of one of the two faces composing the hybrid, as shown by our behavioral data: happy expressions bias the judgment toward the frequency bands in which these are conveyed (see Figure 3), suggesting that gender discrimination itself might actually be combined with a parallel and automatic extraction of the emotional information from the face, including its valence (Vuilleumier, 2007; Vuilleumier and Righart, 2011). In Controls, the increase of activity in the fusiform gyrus when either frequency band conveyed an emotional expression might be a neural signature of such extraction.

In the ASD group, no increase of neural activity was associated with HSF emotional expressions during active gender discrimination, suggesting lower use of emotional information from HSF in this condition, or alternatively increased efficiency at ignoring task-irrelevant information from one specific frequency band. In this perspective, one might expect ASD individuals to be conversely more biased toward LSFs than Controls in their judgments on hybrid faces, a pattern also suggested by visual inspection of behavioral data in Figure 3. Unfortunately, group differences in these behavioral results did not reach statistical significance. In any case, the reported differences in brain activation cannot merely be explained by performance, as LSF- and HSF-biased trials were modeled independently in each participant, and both weighted equally on all subsequent analytical stages regardless of individual idiosyncratic response-biases. We are therefore confident that our results truly reflect differences in visual perceptual processing.

\section{GLOBAL AND LOCAL PROCESSING IN ASD}

At a first sight, ASD's decreased sensitivity to high-frequency information (only during the discrimination task) might be considered at odds with a large body of evidence suggesting how ASD processing of visual stimuli might be biased in favor of detailed (fine-grain) information, at the expense of the global picture. Indeed, ASD individuals have been reported to be more proficient than Controls in tasks in which the global information conflicts with locally-displayed targets (Shah and Frith, 1983; Happé, 1996; Pellicano et al., 2005; Simmons et al., 2009; Almeida et al., 2013) but, at the same time, less proficient in detecting coherent global patterns when intermingled with distracting local information (Spencer et al., 2000; Milne et al., 2002; Pellicano et al., 2005; Spencer and O'Brien, 2006; Tsermentseli et al., 2008).
Please note, however, that the distinction between local vs. global information from earlier studies is not necessarily equivalent to a distinction between HSF vs. LSF information. Indeed, whereas local information is indubitably conveyed by HSF, global information can, at least in principle, be obtained by all frequency ranges, with some critical differences: on the one side, LSF provides global cues from visual stimuli (e.g., a face) regardless of local information, instead HSF can provide global cues by integrating multiple local details together. In this perspective, our findings of decreased HSF-related activity in ASD can be reconciled with earlier accounts only under the assumption that ASD local biases are reflective of a difficulty in seeing the whole through the integration of many details. Consistently with this assumption, recent studies investigated visual integration by using two independent kinds of stimuli: (1) stimuli whose global properties are retained regardless of the details (hierarchical figures, Navon, 1977), for which ASD individuals perform comparably to Controls Deruelle et al., 2006; Rondan and Deruelle, 2007; (2) stimuli whose global properties are retained only from the combined information of many local features (e.g., gestalt illusions of similarity, proximity, etc.), for which ASD individuals exhibit difficulties relative to Controls (Brosnan et al., 2004; Deruelle et al., 2006; Bölte et al., 2007; Rondan and Deruelle, 2007). Please note that in the former kind of stimuli, the global information was available at a coarse level of resolution, thus retainable even after low-pass spatial filtering. This is not necessarily the case for the latter kind of stimuli, in which the global information may also be obtained from information at a more fine-grain level (see also Dakin and Frith, 2005; Simmons et al., 2009 for similar arguments in contour integration tasks).

\section{FUSIFORM AND AMYGDALA FUNCTION IN ASD}

Although many behavioral studies failed at documenting differences in face processing between ASD individuals and Controls, more systematic effects were reported by fMRI studies including reduced neural responses in the fusiform gyrus and the amygdala when processing (emotional or neutral) facial expressions (Baron-Cohen et al., 2000; Critchley et al., 2000; Schultz et al., 2000; Pierce et al., 2001; Hall et al., 2003; Hubl et al., 2003; Wang et al., 2004; Grelotti et al., 2005; Ashwin et al., 2007; Scherf et al., 2010). These results were first interpreted as ASD being characterized by an atypical development of the fusiform gyrus and/or the amygdala (e.g., Baron-Cohen et al., 2000; Schultz, 2005). However, as for other accounts that attempt to describe ASD symptomatology with the dysfunction of specific brain regions (e.g., the broken mirror hypothesis, Hamilton, 2013), these anatomical models are subjected to several critiques. First, some processes associated with the incriminated regions are often spared in ASD individuals (e.g., amygdala dysfunction should also impair emotional arousal, aversive conditioning, or reward contingency learning, but these impairments were not consistently found across studies testing ASD individuals; Gaigg, 2012; see also Zalla and Sperduti, 2013). Second, lesions in incriminated regions, even when occurring at early stages of life, do not lead to the same symptomatology of ASD (Amaral et al., 2003; Paul et al., 2010, but see Bachevalier, 1994). Third, recent studies often report comparable functional properties in 
the incriminated regions between ASD individuals and neurotypical Controls, when controlling for factors such attentional load, stimuli presentation time or eye movements (Hadjikhani et al., 2004, 2007; Dalton et al., 2005; Bird et al., 2006). In this perspective, ASD might not be associated with damaged fusiform gyrus or amygdala per se, but with atypical recruitment/modulation of these regions by high-order top-down control or attentional processes (Santos et al., 2008). Also in our ASD sample the fusiform gyrus and the amygdala did not appear to be generally impaired, e.g., due to either a regional dysfunction or a general atypicality in gazing behavior-but rather this group exhibited a selective hypoactivation for a specific class of information (HSF emotional expressions in hybrid images) and under specific task demands (gender discrimination).

Moreover, Kleinhans et al. (2008) reported decreased functional connectivity between fusiform gyrus and amygdala when ASD participants processed face stimuli, pointing to a dysfunction at the network level rather than at each of its constituent nodes. We concur with this interpretation, but also extend it by offering further insights on the nature of the dysfunction. As shown in Figure 1, the amygdala is thought to receive coarse (LSF) facial information from a direct subcortical (i.e., collicularpulvinar) path, which may then project back to the fusiform (Winston et al., 2003b), whereas in addition the fusiform gyrus also receives fine-grained (HSF) information from a feedforward (i.e., geniculo-striate and ventral occipitotemporal) cortical path. Critically, cortical and subcortical processing of faces are integrated with each other, as shown by enhanced functional connectivity between amygdala and fusiform gyrus during face processing (Morris et al., 1998), and by the impact of amygdala damage on fusiform sensitivity to facial emotional expressions (Vuilleumier et al., 2004). Thus, within this model, we can distinguish between two independent components of the amygdala-fusiform connectivity according to the direction of the information flow. Signaling from the amygdala to the fusiform gyrus is supported by the modulation of fusiform responses by LSF facial information initially processed in the amygdala (see Figure 4A, but also Vuilleumier et al., 2003; Winston et al., 2003b). Conversely, signaling from the fusiform to the amygdala is consistent with amygdala responses being also sensitive to HSF facial information conveyed by the visual cortex (see Figure 5). Our data provide novel evidence suggesting that it is the signal in the latter (but not the former) direction that exhibits atypical properties by ASD. This in turn suggests that integrative face processing functions mediated by higher level visual cortices might be more affected by ASD than lower level subcortical pathways providing inputs to the amygdala.

\section{LIMITATIONS OF THE STUDY AND FUTURE RESEARCH}

Like many other neuroimaging investigations on autism, including those reviewed in this article, our dataset is penalized by the limited number of participants and by an ASD population including both individuals affected by Asperger Syndrome and High Functioning Autism (see Table 1). Low power is not necessarily detrimental for positive results, which in our case were all obtained under corrected statistical thresholds (see also Friston, 2012), but it is problematic for those tests producing null or marginal results and for which an effect could potentially still be found with a larger sample. Also the heterogeneity of the clinical sample might be an additional source of noise with detrimental effects on the power of statistical analysis. Furthermore, some of the effects might be driven by only one of the two clinical sub-groups without a possibility of further verification on corresponding subsamples. It should be stressed, however, that the distinction between Asperger Syndrome and High Functioning Autism was removed in the last edition of the Diagnostic and Statistical Manual of mental disorders (American Psychiatric Association, 2013). In this perspective, putative heterogeneities in our clinical population should be treated as any within-group variability against which the significance of effects is estimated.

In particular, low power and sample heterogeneity might account for the weak effects of valence of emotional face expressions. Indeed, participants' behavior in both groups was significantly affected by valence, while the analysis of the fMRI signal did not reveal a similar effect in the brain. We should stress, however, that this consideration is not critical for our main results, since a lack of valence effects in the fusiform gyrus and the amygdala is plausible with respect to earlier accounts (Sander et al., 2003; Surguladze et al., 2003; Winston et al., 2003a). Interestingly, however, during the passive task, ASD individuals exhibited increased activity for LSF happy as opposed to LSF fearful expressions in the right amygdala. This activation arose in a ventrolateral portion of the amygdala, whereas earlier effects associated with the discrimination task arose in a more dorsal and medial location (Figure 8B). Parcellation of the human amygdala has been carried out with both cytoarchitectonic (Amunts et al., 2005) and connectivity-based approaches (Bzdok et al., 2013), and suggest that the different effects in Figure 8B might concern different sub-regions. Future research with high-resolution fMRI techniques is needed to investigate more specifically how ASD impairments in face and emotional processing might relate to different subregions of the amygdala.

Furthermore, caution should be used to interpret group differences in their response to LSF expressions relative to the neutral control condition because, unlike for HSFs, no significant interaction with the group factor was found. We can therefore not conclude whether the lateralization displayed in Figure 5A is truly reflective of different network-organization in the two groups. Please notice that, although left FFA was identified only when testing ASD individuals, visual inspection of the parameters extracted from this region suggests that a similar effect might be present also in Controls. It is therefore plausible to assume that, like for HSFs, the greater sensitivity of Controls to LSF expressions might extend to both hemispheres.

Finally, although we are quite confident that in the gender discrimination task participants focused their attention on global aspects of the face stimuli (Zhao and Hayward, 2010), we have little control on which processes were at play during the passive viewing session, in which the only instruction was to watch the stimuli attentively. Furthermore, even if participants focused on face stimuli, we do not know whether they preferentially attended to global or local properties or shifted between both. In this perspective, the increase of neural activity observed in ASD individuals for HSF emotional information in the passive condition 
(Figure 8) can only be taken as evidence for spared functionality of the cortical path outside the demands of the discrimination task (see Discussion section above). Future studies will need to extend these results by using other tasks in which participants are forced to focus on local facial details, thus allowing us to determine the neural signatures associated with featural facial processing in addition to the frequency content manipulation used here.

\section{REFERENCES}

Almeida, R. A., Dickinson, J. E., Maybery, M. T., Badcock, J. C., and Badcock, D. R. (2013). Visual search targeting either local or global perceptual processes differs as a function of autistic-like traits in the typically developing population. J. Autism Dev. Disord. 43, 1272-1286. doi: 10.1007/s10803-012-1669-7

Amaral, D. G., Bauman, M. D., and Schumann, C. M. (2003). The amygdala and autism: implications from non-human primate studies. Genes Brain Behav. 2, 295-302. doi: 10.1034/j.1601-183X.2003.00043.x

American Psychiatric Association. (2013). Diagnostic and Statistical Manual of Mental Disorders: DSM-5. Arlington, VA: American Psychiatric Association.

Amunts, K., Kedo, O., Kindler, M., Pieperhoff, P., Mohlberg, H., Shah, N. J., et al. (2005). Cytoarchitectonic mapping of the human amygdala, hippocampal region and entorhinal cortex: intersubject variability and probability maps. Anat. Embryol. 210, 343-352. doi: 10.1007/s00429-005-0025-5

Ashwin, C., Baron-Cohen, S., Wheelwright, S., O'Riordan. M., and Bullmore, E. T. (2007). Differential activation of the amygdala and the "social brain" during fearful face-processing in Asperger Syndrome. Neuropsychologia 45, 2-14. doi: 10.1016/j.neuropsychologia.2006.04.014

Bachevalier, J. (1994). Medial temporal lobe structures and autism: a review of clinical and experimental findings. Neuropsychologia 32, 627-648. doi: 10.1016/0028-3932(94)90025-6

Baron-Cohen, S., Ring, H. A., Bullmore, E. T., Wheelwright, S., Ashwin, C., and Williams, S. C. R. (2000). The amygdala theory of autism. Neurosci. Biobehav. Rev. 24, 355-364. doi: 10.1016/S0149-7634(00)00011-7

Barton, J. J. S., Hefter, R. L., Cherkasova, M. V., and Manoach, D. S. (2007). Investigations of face expertise in the social developmental disorders. Neurology 69, 860-870. doi: 10.1212/01.wnl.0000267842.85646.f2

Bertone, A., Mottron, L., Jelenic, P., and Faubert, J. (2005). Enhanced and diminished visuo-spatial information processing in autism depends on stimulus complexity. Brain 128, 2430-2441. doi: 10.1093/brain/awh561

Bird, G., Catmur, C., Silani, G., Frith, C., and Frith, U. (2006). Attention does not modulate neural responses to social stimuli in autism spectrum disorders. Neuroimage 31, 1614-1624. doi: 10.1016/j.neuroimage.2006.02.037

Boeschoten, M. A., Kenemans, J. L., van Engeland, H., and Kemner, C. (2007b). Abnormal spatial frequency processing in high-functioning children with pervasive developmental disorder (PDD). Clin. Neurophysiol. 118, 2076-2088. doi: 10.1016/j.clinph.2007.05.004

Boeschoten, M. A., Kenemans, J. L., van Engeland, H., and Kemner, C. (2007a). Face processing in Pervasive Developmental Disorder (PDD): the roles of expertise and spatial frequency. J Neural Transm. 114, 1619-1629. doi: 10.1007/s00702007-0780-y

Bölte, S., Holtmann, M., Poustka, F., Scheurich, A., and Schmidt, L. (2007). Gestalt perception and local-global processing in high-functioning autism. J. Autism Dev. Disord. 37, 1493-1504. doi: 10.1007/s10803-006-0231-x

Boutet, I., Collin, C., and Faubert, J. (2003). Configural face encoding and spatial frequency information. Percept. Psychophys 65, 1078-1093. doi: 10.3758/BF03194835

Brosnan, M. J., Scott, F. J., Fox, S., and Pye, J. (2004). Gestalt processing in autism: failure to process perceptual relationships and the implications for contextual understanding. J. Child Psychol. Psychiatry 45, 459-469. doi: 10.1111/j.14697610.2004.00237.x

Bzdok, D., Laird, A. R., Zilles, K., Fox, P. T., and Eickhoff, S. B. (2013). An investigation of the structural, connectional, and functional subspecialization in the human amygdala. Hum. Brain Mapp. 34, 3247-3266. doi: 10.1002/hbm.22138

Carretié, L., Hinojosa, J. A., López-Martín, S., and Tapia, M. (2007). An electrophysiological study on the interaction between emotional content and spatial frequency of visual stimuli. Neuropsychologia 45, 1187-1195. doi: 10.1016/j.neuropsychologia.2006.10.013
Collin, C. A., Therrien, M., Martin, C., and Rainville, S. (2006). Spatial frequency thresholds for face recognition when comparison faces are filtered and unfiltered. Percept. Psychophys. 68, 879-889. doi: 10.3758/BF03193351

Costen, N. P., Parker, D. M., and Craw, I. (1994). Spatial content and spatial quantisation effects in face recognition. Perception 23, 129-146. doi: 10.1068/p230129

Costen, N. P., Parker, D. M., and Craw, I. (1996). Effects of high-pass and lowpass spatial filtering on face identification. Percept. Psychophys. 58, 602-612. doi: 10.3758/BF03213093

Critchley, H. D., Daly, E. M., Bullmore, E. T., Williams, S. C. R., Amelsvoort, T. V., Robertson, D. M., et al. (2000). The functional neuroanatomy of social behaviour Changes in cerebral blood flow when people with autistic disorder process facial expressions. Brain 123, 2203-2212. doi: 10.1093/brain/123.11.2203

Dakin, S., and Frith, U. (2005). Vagaries of visual perception in autism. Neuron 48, 497-507. doi: 10.1016/j.neuron.2005.10.018

Dalton, K. M., Nacewicz, B. M., Johnstone, T., Schaefer, H. S., Gernsbacher, M. A., Goldsmith, H. H., et al. (2005). Gaze fixation and the neural circuitry of face processing in autism. Nat. Neurosci. 8, 519-526. doi: 10.1038/nn1421

Davis, R. A. O., Bockbrader, M. A., Murphy, R. R., Hetrick, W. P., and O’Donnell, B. F. (2006). Subjective perceptual distortions and visual dysfunction in children with autism. J. Autism Dev. Disord. 36, 199-210. doi: 10.1007/s10803-0050055-0

De Gelder, B., Vroomen, J., Pourtois, G., and Weiskrantz, L. (1999). Non-conscious recognition of affect in the absence of striate cortex. Neuroreport 10, 3759-3763. doi: 10.1097/00001756-199912160-00007

De Jonge, M. V., Kemner, C., de Haan, E. H., Coppens, J. E., van den Berg, T. J. T. P., and van Engeland, H. (2007). Visual information processing in high-functioning individuals with autism spectrum disorders and their parents. Neuropsychology 21, 65-73. doi: 10.1037/0894-4105.21.1.65

Deruelle, C., Rondan, C., Gepner, B., and Fagot, J. (2006). Processing of compound visual stimuli by children with autism and Asperger syndrome. Int. J. Psychol. 41, 97-106. doi: 10.1080/00207590500184610

Deruelle, C., Rondan, C., Gepner, B., and Tardif, C. (2004). Spatial frequency and face processing in children with autism and Asperger syndrome. J. Autism Dev. Disord. 34, 199-210. doi: 10.1023/B:JADD.0000022610.09668.4c

Deruelle, C., Rondan, C., Salle-Collemiche, X., Bastard-Rosset, D., and Da Fonséca, D. (2008). Attention to low- and high-spatial frequencies in categorizing facial identities, emotions and gender in children with autism. Brain Cogn. 66, 115-123. doi: 10.1016/j.bandc.2007.06.001

Dickstein, D. P., Pescosolido, M. F., Reidy, B. L., Galvan, T., Kim, K. L., Seymour, K. E., et al. (2013). Developmental meta-analysis of the functional neural correlates of autism spectrum disorders. J. Am. Acad. Child. Adolesc. Psychiatry 52, 279-289.e16. doi: 10.1016/j.jaac.2012.12.012

Friston, K. (2012). Ten ironic rules for non-statistical reviewers. Neuroimage 61, 1300-1310. doi: 10.1016/j.neuroimage.2012.04.018

Friston, K. J., Worsley, K. J., Frackowiak, R. S. J., Mazziotta, J. C., and Evans, A. C. (1993). Assessing the significance of focal activations using their spatial extent. Hum. Brain Mapp. 1, 210-220. doi: 10.1002/hbm.460010306

Gaigg, S. B. (2012). The interplay between emotion and cognition in autism spectrum disorder: implications for developmental theory. Front. Integr. Neurosci. 6:113. doi: 10.3389/fnint.2012.00113

Goeleven, E., De Raedt, R., Leyman, L., and Verschuere, B. (2008). The karolinska directed emotional faces: a validation study. Cogn. Emot. 22, 1094-1118. doi: $10.1080 / 02699930701626582$

Gold, J., Bennett, P. J., and Sekuler, A. B. (1999). Identification of band-pass filtered letters and faces by human and ideal observers. Vision Res. 39, 3537-3560. doi: 10.1016/S0042-6989(99)00080-2

Grelotti, D. J., Klin, A. J., Gauthier, I., Skudlarski, P., Cohen, D. J., Gore, J. C., et al. (2005). fMRI activation of the fusiform gyrus and amygdala to cartoon characters but not to faces in a boy with autism. Neuropsychologia 43, 373-385. doi: 10.1016/j.neuropsychologia.2004.06.015

Gross, T. F. (2008). Recognition of immaturity and emotional expressions in blended faces by children with autism and other developmental disabilities. J. Autism Dev. Disord. 38, 297-311. doi: 10.1007/s10803-007-0391-3

Gschwind, M., Pourtois, G., Schwartz, S., Van De Ville, D., and Vuilleumier, P. (2012). White-matter connectivity between face-responsive regions in the human brain. Cereb. Cortex 22, 1564-1576. doi: 10.1093/cercor/bhr226

Hadjikhani, N., Joseph, R. M., Snyder, J., Chabris, C. F., Clark, J., Steele, S., et al. (2004). Activation of the fusiform gyrus when individuals with 
autism spectrum disorder view faces. Neuroimage 22, 1141-1150. doi: 10.1016/j.neuroimage.2004.03.025

Hadjikhani, N., Joseph, R. M., Snyder, J., and Tager-Flusberg, H. (2007). Abnormal activation of the social brain during face perception in autism. Hum. Brain Mapp. 28, 441-449. doi: 10.1002/hbm.20283

Hall, G. B., Szechtman, H., and Nahmias, C. (2003). Enhanced salience and emotion recognition in autism: A, P. E. T. study. Am. J. Psychiatry 160, 1439-1441. doi: 10.1176/appi.ajp.160.8.1439

Hamilton, A. F. (2013). Reflecting on the mirror neuron system in autism: a systematic review of current theories. Dev. Cogn. Neurosci. 3, 91-105. doi: 10.1016/j.dcn.2012.09.008

Happé, F., and Frith, U. (2006). The weak coherence account: detail-focused cognitive style in autism spectrum disorders. J. Autism Dev. Disord. 36, 5-25. doi: 10.1007/s10803-005-0039-0

Happé, F. G. E. (1996). Studying weak central coherence at low levels: children with autism do not succumb to visual illusions. A research note. J. Child. Psychol. Psychiatry 37, 873-877. doi: 10.1111/j.1469-7610.1996.tb01483.x

Harms, M. B., Martin, A., and Wallace, G. L. (2010). Facial emotion recognition in autism spectrum disorders: a review of behavioral and neuroimaging studies. Neuropsychol. Rev. 20, 290-322. doi: 10.1007/s11065-010-9138-6

Haxby, J. V., Hoffman, E. A., and Gobbini, M. I. (2000). The distributed human neural system for face perception. Trends Cogn. Sci. 4, 223-233. doi: 10.1016/S1364-6613(00)01482-0

Hobson, R. P., Ouston, J., and Lee, A. (1988). What's in a face? The case of autism. Br. J. Psychol. 79, 441-453. doi: 10.1111/j.2044-8295.1988.tb02745.x

Hubl, D., Bölte, S., Feineis-Matthews, S., Lanfermann, H., Federspiel, A., Strik, W., et al. (2003). Functional imbalance of visual pathways indicates alternative face processing strategies in autism. Neurology 61, 1232-1237. doi: 10.1212/01.WNL.0000091862.22033.1A

Joseph, R. M., and Tanaka, J. (2003). Holistic and part-based face recognition in children with autism. J. Child Psychol. Psychiatry 44, 529-542. doi: 10.1111/1469-7610.00142

Kanwisher, N., McDermott, J., and Chun, M. M. (1997). The fusiform face area: a module in human extrastriate cortex specialized for face perception. J Neurosci. 17, 4302-4311.

Kleinhans, N. M., Richards, T., Sterling, L., Stegbauer, K. C., Mahurin, R., Johnson, L. C., et al. (2008). Abnormal functional connectivity in autism spectrum disorders during face processing. Brain J. Neurol. 131, 1000-1012. doi: 10.1093/brain/awm334

Lahaie, A., Mottron, L., Arguin, M., Berthiaume, C., Jemel, B., and Saumier, D. (2006). Face perception in high-functioning autistic adults: evidence for superior processing of face parts, not for a configural face-processing deficit. Neuropsychology 20, 30-41. doi: 10.1037/0894-4105.20.1.30

Livingstone, M., and Hubel, D. (1988). Segregation of form, color, movement, and depth: anatomy, physiology, and perception. Science 240, 740-749. doi: 10.1126/science.3283936

Livingstone, M. S., and Hubel, D. H. (1987). Psychophysical evidence for separate channels for the perception of form, color, movement, and depth. J. Neurosci. 7, 3416-3468.

López, B., Donnelly, N., Hadwin, J., and Leekam, S. (2004). Face processing in highfunctioning adolescents with autism: evidence for weak central coherence. Vis. Cogn. 11, 673-688. doi: 10.1080/13506280344000437

Lord, C., Risi, S., Lambrecht, L., Cook, E. H. Jr., Leventhal, B. L., DiLavore, P. C., et al. (2000). The autism diagnostic observation schedule-generic: a standard measure of social and communication deficits associated with the spectrum of autism. J. Autism Dev. Disord. 30, 205-223. doi: 10.1023/A:1005592401947

Lord, C., Rutter, M., and Le Couteur, A. (1994). Autism Diagnostic InterviewRevised: a revised version of a diagnostic interview for caregivers of individuals with possible pervasive developmental disorders. J. Autism Dev. Disord. 24, 659-685. doi: 10.1007/BF02172145

Milne, E., Scope, A., Pascalis, O., Buckley, D., and Makeig, S. (2009). Independent component analysis reveals atypical electroencephalographic activity during visual perception in individuals with autism. Biol. Psychiatry 65, 22-30. doi: 10.1016/j.biopsych.2008.07.017

Milne, E., Swettenham, J., Hansen, P., Campbell, R., Jeffries, H., and Plaisted, K. (2002). High motion coherence thresholds in children with autism. J. Child Psychol. Psychiatry 43, 255-263. doi: 10.1111/1469-7610.00018

Morris, J. S., Friston, K. J., Büchel, C., Frith, C. D., Young, A. W., Calder, A. J., et al. (1998). A neuromodulatory role for the human amygdala in processing emotional facial expressions. Brain J. Neurol. 121(Pt 1), 47-57. doi: 10.1093/ brain/121.1.47

Morris, J. S., Öhman, A., and Dolan, R. J. (1999). A subcortical pathway to the right amygdala mediating "unseen" fear. Proc. Natl. Acad. Sci. U.S.A. 96, 1680-1685. doi: $10.1073 /$ pnas.96.4.1680

Mottron, L., Dawson, M., Soulières, I., Hubert, B., and Burack, J. (2006). Enhanced perceptual functioning in autism: an update, and eight principles of autistic perception. J. Autism Dev. Disord. 36, 27-43. doi: 10.1007/s10803-0050040-7

Näsänen, R. (1999). Spatial frequency bandwidth used in the recognition of facial images. Vision Res. 39, 3824-3833. doi: 10.1016/S0042-6989(99)00096-6

Navon, D. (1977). Forest before trees: the precedence of global features in visual perception. Cognit Psychol. 9, 353-383. doi: 10.1016/0010-0285(77)90012-3

Oldfield, R. C. (1971). The assessment and analysis of handedness: the Edinburgh inventory. Neuropsychologia 9, 97-113. doi: 10.1016/0028-3932(71)90067-4

Parker, D. M., and Costen, N. P. (1999). One extreme or the other or perhaps the golden mean? Issues of spatial resolution in face processing. Curr. Psychol. 18, 118-127. doi: 10.1007/s12144-999-1021-3

Paul, L. K., Corsello, C., Tranel, D., and Adolphs, R. (2010). Does bilateral damage to the human amygdala produce autistic symptoms? J. Neurodev. Disord. 2, 165-173. doi: 10.1007/s11689-010-9056-1

Pellicano, E., Gibson, L., Maybery, M., Durkin, K., and Badcock, D. R. (2005). Abnormal global processing along the dorsal visual pathway in autism: a possible mechanism for weak visuospatial coherence? Neuropsychologia 43, 1044-1053. doi: 10.1016/j.neuropsychologia.2004.10.003

Pellicano, E., Jeffery, L., Burr, D., and Rhodes, G. (2007). Abnormal adaptive facecoding mechanisms in children with autism spectrum disorder. Curr. Biol. 17, 1508-1512. doi: 10.1016/j.cub.2007.07.065

Penny, W. D., and Holmes, A. P. (2004). "Random effects analysis," in Human Brain Function, 2nd Edn, eds R. S. J. Frackowiak, J. T. Ashburner, W. D. Penny, and S. Zeki (Academic Press), 843-850.

Pessoa, L., and Adolphs, R. (2010). Emotion processing and the amygdala: from a "low road" to "many roads" of evaluating biological significance. Nat. Rev. Neurosci. 11, 773-783. doi: 10.1038/nrn2920

Pierce, K., Müller, R. A., Ambrose, J., Allen, G., and Courchesne, E. (2001). Face processing occurs outside the fusiform "face area" in autism: evidence from functional MRI. Brain J Neurol. 124, 2059-2073. doi: 10.1093/brain/124. 10.2059

Pourtois, G., Dan, E. S., Grandjean, D., Sander, D., and Vuilleumier, P. (2005). Enhanced extrastriate visual response to bandpass spatial frequency filtered fearful faces: time course and topographic evokedpotentials mapping. Hum. Brain Mapp. 26, 65-79. doi: 10.1002/ hbm. 20130

Riby, D. M., Doherty-Sneddon, G., and Bruce, V. (2009). The eyes or the mouth? Feature salience and unfamiliar face processing in Williams syndrome and autism. Q. J. Exp. Psychol. 2006. 62, 189-203. doi: 10.1080/17470210701855629

Rondan, C., and Deruelle, C. (2004). Face processing in high functioning autistic adults: a look into spatial frequencies and the inversion effect. J. Cogn. Behav. Psychother. 4, 149-163.

Rondan, C., and Deruelle, C. (2007). Global and configural visual processing in adults with autism and Asperger syndrome. Res. Dev. Disabil. 28, 197-206. doi: 10.1016/j.ridd.2006.02.007

Rotshtein, P., Vuilleumier, P., Winston, J., Driver, J., and Dolan, R. (2007). Distinct and convergent visual processing of high and low spatial frequency information in faces. Cereb. Cortex 17, 2713-2724. doi: 10.1093/cercor/bhl180

Rouse, H., Donnelly, N., Hadwin, J. A., and Brown, T. (2004). Do children with autism perceive second-order relational features? The case of the Thatcher illusion. J. Child. Psychol. Psychiatry 45, 1246-1257. doi: 10.1111/j.14697610.2004.00317.x

Sander, D., Grafman, J., and Zalla, T. (2003). The human amygdala: an evolved system for relevance detection. Rev. Neurosci. 14, 303-316. doi: 10.1515/REVNEURO.2003.14.4.303

Santos, A., Rondan, C., Rosset, D. B., Fonseca, D. D., and Deruelle, C. (2008) Mr. Grimace or Ms. Smile does categorization affect perceptual processing in autism? Psychol Sci. 19, 70-76. doi: 10.1111/j.1467-9280.2008.02048.x

Scherf, K. S., Luna, B., Minshew, N., and Behrmann, M. (2010). Location, location, location: alterations in the functional topography of face- but not object- or place-related cortex in adolescents with autism. Front. Hum. Neurosci. 4:26. doi: 10.3389/fnhum.2010.00026 
Schultz, R. T. (2005). Developmental deficits in social perception in autism: the role of the amygdala and fusiform face area. Int. J. Dev. Neurosci. 23, 125-141. doi: 10.1016/j.ijdevneu.2004.12.012

Schultz, R. T., Gauthier, I., Klin, A., Fulbright, R. K., Anderson, A. W., Volkmar, F., et al. (2000). Abnormal ventral temporal cortical activity during face discrimination among individuals with autism and Asperger syndrome. Arch. Gen. Psychiatry 57, 331-340. doi: 10.1001/archpsyc.57.4.331

Schwarzlose, R. F., Baker, C. I., and Kanwisher, N. (2005). Separate face and body selectivity on the fusiform gyrus. J. Neurosci. 25, 11055-11059. doi: 10.1523/JNEUROSCI.2621-05.2005

Schyns, P. G., and Oliva, A. (1999). Dr. Angry and Mr. Smile: when categorization flexibly modifies the perception of faces in rapid visual presentations. Cognition 69, 243-265. doi: 10.1016/S0010-0277(98)00069-9

Shah, A., and Frith, U. (1983). An islet of ability in autistic children: a research note. J. Child Psychol. Psychiatry 24, 613-620. doi: 10.1111/j.14697610.1983.tb00137.x

Simmons, D. R., Robertson, A. E., McKay, L. S., Toal, E., McAleer, P., and Pollick, F. E. (2009). Vision in autism spectrum disorders. Vision Res. 49, 2705-2739. doi: 10.1016/j.visres.2009.08.005

Spencer, J., O’Brien, J., Riggs, K., Braddick, O., Atkinson, J., and WattamBell, J. (2000). Motion processing in autism: evidence for a dorsal stream deficiency. Neuroreport 11, 2765-2767. doi: 10.1097/00001756-20000821000031

Spencer, J. V., and O'Brien, J. M. D. (2006). Visual form-processing deficits in autism. Perception 35, 1047-1055. doi: 10.1068/p5328

Spiridon, M., Fischl, B., and Kanwisher, N. (2006). Location and spatial profile of category-specific regions in human extrastriate cortex. Hum. Brain Mapp. 27, 77-89. doi: 10.1002/hbm.20169

Surguladze, S. A., Brammer, M. J., Young, A. W., Andrew, C., Travis, M. J., Williams, S. C., et al. (2003). A preferential increase in the extrastriate response to signals of danger. Neuroimage 19, 1317-1328. doi: 10.1016/S1053-8119(03) 00085-5

Tanaka, J. W., and Farah, M. J. (1993). Parts and wholes in face recognition. Q. J. Exp. Psychol. A 46, 225-245. doi: 10.1080/14640749308401045

Teunisse, J.-P., and de Gelder, B. (2003). Face processing in adolescents with autistic disorder: the inversion and composite effects. Brain Cogn. 52, 285-294. doi: 10.1016/S0278-2626(03)00042-3

Tsermentseli, S., O'Brien, J. M., and Spencer, J. V. (2008). Comparison of form and motion coherence processing in autistic spectrum disorders and dyslexia. J. Autism Dev. Disord. 38, 1201-1210. doi: 10.1007/s10803-007-0500-3

Tzourio-Mazoyer, N., Landeau, B., Papathanassiou, D., Crivello, F., Etard, O., Delcroix, N., et al. (2002). Automated anatomical labeling of activations in SPM using a macroscopic anatomical parcellation of the MNI MRI single-subject brain. Neuroimage 15, 273-289. doi: 10.1006/nimg.2001.0978

Valentine, T. (1988). Upside-down faces: a review of the effect of inversion upon face recognition. Br. J. Psychol. 79, 471-491. doi: 10.1111/j.20448295.1988.tb02747.x

Van Der Geest, J. N., Kemner, C., Verbaten, M. N., and Van Engeland, H. (2002). Gaze behavior of children with pervasive developmental disorder toward human faces: a fixation time study. J. Child Psychol. Psychiatry 43, 669-678. doi: 10.1111/1469-7610.00055

Vlamings, P. H. J. M., Jonkman, L. M., van Daalen, E., van der Gaag, R. J., and Kemner, C. (2010). Basic abnormalities in visual processing affect face processing at an early age in autism spectrum disorder. Biol. Psychiatry 68, 1107-1113. doi: 10.1016/j.biopsych.2010.06.024

Vuilleumier, P. (2007). "Neural representation of faces in human visual cortex: the roles of attention, emotion, and viewpoint," in Object Recognition, Attention, and Action, eds N. Osaka, I. Rentschler, and I. Biederman (Springer Japan), 119-138. doi: 10.1007/978-4-431-73019-4_9
Vuilleumier, P., Armony, J. L., Driver, J., and Dolan, R. J. (2001). Effects of attention and emotion on face processing in the human brain: an event-related fMRI study. Neuron 30, 829-841. doi: 10.1016/S0896-6273(01)00328-2

Vuilleumier, P., Armony, J. L., Driver, J., and Dolan, R. J. (2003). Distinct spatial frequency sensitivities for processing faces and emotional expressions. Nat. Neurosci. 6, 624-631. doi: 10.1038/nn1057

Vuilleumier, P., and Pourtois, G. (2007). Distributed and interactive brain mechanisms during emotion face perception: evidence from functional neuroimaging. Neuropsychologia 45, 174-194. doi: 10.1016/j.neuropsychologia.2006.06.003

Vuilleumier, P., Richardson, M. P., Armony, J. L., Driver, J., and Dolan, R. J. (2004). Distant influences of amygdala lesion on visual cortical activation during emotional face processing. Nat. Neurosci. 7, 1271-1278. doi: 10.1038/nn1341

Vuilleumier, P., and Righart, R. (2011). "Attention and automaticity in processing facial expressions," in Oxford Handbook of Face Perception, eds A. Calder, G. Rhodes, M. Johnson, and J. Haxby (New York, NY: Oxford University Press), 449-478. doi: 10.1093/oxfordhb/9780199559053.013.0023

Wang, A. T., Dapretto, M., Hariri, A. R., Sigman, M., and Bookheimer, S. Y. (2004). Neural correlates of facial affect processing in children and adolescents with autism spectrum disorder. J. Am. Acad. Child Adolesc. Psychiatry 43, 481-490. doi: 10.1097/00004583-200404000-00015

Watier, N. N., Collin, C. A., and Boutet, I. (2010). Spatial-frequency thresholds for configural and featural discriminations in upright and inverted faces. Perception 39, 502-513. doi: 10.1068/p6504

Weigelt, S., Koldewyn, K., and Kanwisher, N. (2012). Face identity recognition in autism spectrum disorders: a review of behavioral studies. Neurosci. Biobehav. Rev. 36, 1060-1084. doi: 10.1016/j.neubiorev.2011.12.008

Winston, J., O'Doherty, J., and Dolan, R. (2003a). Common and distinct neural responses during direct and incidental processing of multiple facial emotions. Neuroimage 20, 84-97. doi: 10.1016/S1053-8119(03)00303-3

Winston, J. S., Vuilleumier, P., and Dolan, R. J. (2003b). Effects of low-spatial frequency components of fearful faces on fusiform cortex activity. Curr. Biol. 13, 1824-1829. doi: 10.1016/j.cub.2003.09.038

Young, A. W., Hellawell, D., and Hay, D. C. (1987). Configurational information in face perception. Perception 16, 747-759. doi: 10.1068/p160747

Zalla, T., and Sperduti, M. (2013). The amygdala and the relevance detection theory of autism: an evolutionary perspective. Front. Hum. Neurosci. 7:894. doi: 10.3389/fnhum.2013.00894

Zhao, M., and Hayward, W. G. (2010). Holistic processing underlies gender judgments of faces. Atten. Percept. Psychophys 72, 591-596. doi: 10.3758/APP.72.3.591

Conflict of Interest Statement: The authors declare that the research was conducted in the absence of any commercial or financial relationships that could be construed as a potential conflict of interest.

Received: 30 October 2013; paper pending published: 10 December 2013; accepted: 14 March 2014; published online: 09 April 2014.

Citation: Corradi-Dell'Acqua C, Schwartz S, Meaux E, Hubert B, Vuilleumier P and Deruelle $C$ (2014) Neural responses to emotional expression information in highand low-spatial frequency in autism: evidence for a cortical dysfunction. Front. Hum. Neurosci. 8:189. doi: 10.3389/fnhum.2014.00189

This article was submitted to the journal Frontiers in Human Neuroscience.

Copyright (c) 2014 Corradi-Dell'Acqua, Schwartz, Meaux, Hubert, Vuilleumier and Deruelle. This is an open-access article distributed under the terms of the Creative Commons Attribution License (CC BY). The use, distribution or reproduction in other forums is permitted, provided the original author(s) or licensor are credited and that the original publication in this journal is cited, in accordance with accepted academic practice. No use, distribution or reproduction is permitted which does not comply with these terms. 